\title{
The Paradoxical Effect of Creatine Monohydrate on Muscle Damage Markers: A Systematic Review and Meta-Analysis
}

\author{
$K_{\text {Kenji Doma }}^{1}\left[\right.$ (] Akhilesh Kumar Ramachandran ${ }^{2} \cdot$ Daniel Boullosa $^{1,3} \cdot$ Jonathan Connor $^{1}$
}

Accepted: 2 January 2022 / Published online: 26 February 2022

(c) The Author(s) 2022

\begin{abstract}
Background Several studies have examined the effect of creatine monohydrate (CrM) on indirect muscle damage markers and muscle performance, although pooled data from several studies indicate that the benefits of CrM on recovery dynamics are limited.

Objective This systematic review and meta-analysis determined whether the ergogenic effects of CrM ameliorated markers of muscle damage and performance following muscle-damaging exercises.

Methods In total, 23 studies were included, consisting of 240 participants in the CrM group (age $23.9 \pm 10.4$ years, height $178 \pm 5 \mathrm{~cm}$, body mass $76.9 \pm 7.6 \mathrm{~kg}$, females $10.4 \%$ ) and 229 participants in the placebo group (age $23.7 \pm 8.5$ years, height $177 \pm 5 \mathrm{~cm}$, body mass $77.0 \pm 6.6 \mathrm{~kg}$, females $10.0 \%$ ). These studies were rated as fair to excellent following the PEDro scale. The outcome measures were compared between the CrM and placebo groups at $24-36 \mathrm{~h}$ and $48-90 \mathrm{~h}$ following muscledamaging exercises, using standardised mean differences (SMDs) and associated $p$-values via forest plots. Furthermore, sub-group analyses were conducted by separating studies into those that examined the effects of $\mathrm{CrM}$ as an acute training response (i.e., after one muscle-damaging exercise bout) and those that examined the chronic training response (i.e., examining the acute response after the last training session following several weeks of training).

Results According to the meta-analysis, the CrM group exhibited significantly lower indirect muscle damage markers (i.e., creatine kinase, lactate dehydrogenase, and/or myoglobin) at 48-90 h post-exercise for the acute training response (SMD - 1.09; $p=0.03$ ). However, indirect muscle damage markers were significantly greater in the CrM group at $24 \mathrm{~h}$ post-exercise (SMD 0.95; $p=0.04$ ) for the chronic training response. Although not significant, a large difference in indirect muscle damage markers was also found at $48 \mathrm{~h}$ post-exercise (SMD 1.24) for the chronic training response. The CrM group also showed lower inflammation for the acute training response at $24-36 \mathrm{~h}$ post-exercise and $48-90 \mathrm{~h}$ post-exercise with a large effect size (SMD $-1.38 \leq d \leq-1.79$ ). Similarly, the oxidative stress markers were lower for the acute training response in the CrM group at 24-36 h post-exercise and $90 \mathrm{~h}$ post-exercise, with a large effect size (SMD -1.37 and -1.36 , respectively). For delayed-onset muscle soreness (DOMS), the measures were lower for the CrM group at $24 \mathrm{~h}$ post-exercise with a moderate effect size (SMD - 0.66) as an acute training response. However, the inter-group differences for inflammation, oxidative stress, and DOMS were not statistically significant $(p>0.05)$.

Conclusion Overall, our meta-analysis demonstrated a paradoxical effect of CrM supplementation post-exercise, where CrM appears to minimise exercise-induced muscle damage as an acute training response, although this trend is reversed as a chronic training response. Thus, CrM may be effective in reducing the level of exercise-induced muscle damage following a single bout of strenuous exercises, although training-induced stress could be exacerbated following long-term supplementation of CrM. Although long-term usage of CrM is known to enhance training adaptations, whether the increased level of exercise-induced muscle damage as a chronic training response may provide potential mechanisms to enhance chronic training adaptations with CrM supplementation remains to be confirmed.
\end{abstract}

Extended author information available on the last page of the article 


\section{Key Points}

Creatine monohydrate reduced the level of exerciseinduced muscle damage as an acute training response, although this trend was reversed as a chronic training response.

Creatine monohydrate may be an ergogenic supplement to accelerate recovery following a single bout of strenuous exercise.

The increased level of exercise-induced muscle damage after several weeks of training and creatine monohydrate supplementation may suggest a possibly greater tolerance of training stresses, given that long-term creatine monohydrate supplementation is known to enhance training adaptation.

\section{Introduction}

Exercise-induced muscle damage (EIMD) is a common phenomenon following muscular contractions, particularly with unfamiliar activities, eccentric contractions, or those under heavy loads [1]. The common symptoms of EIMD include increased serum muscle proteins (e.g., creatine kinase [CK]), inflammatory markers (e.g., interleukin [IL]-6), oxidative stress (e.g., hydrogen peroxide), delayed onset of muscle soreness (DOMS), and prolonged impairment in functional performance [2-4]. Furthermore, EIMD impairs running [5-7], cycling power output [8], and sprint and agility performances $[9,10]$, which may induce sub-optimal training adaptation if symptoms of EIMD are extended [11]. However, effective dietary supplements may minimise the negative effects of EIMD without compromising long-term adaptations [12], thus reducing the need for resting days and reduced workload to favour appropriate recovery between training bouts.

Several commercially available oral supplements are known to minimise the level of EIMD, including extracts derived from fruits [13], tea leaf [14], and root plants [15], which contain a high amount of antioxidant and antiinflammatory properties. Although these supplements may aid in recovery following strenuous exercise and enhance preparedness for athletes between training bouts, the benefits in terms of enhanced chronic training adaptations are not well documented. On the other hand, creatine monohydrate (CrM) has long been used as an oral supplement to enhance muscular strength [16] and hypertrophic adaptations [17] when ingested during resistance training periods. A number of mechanisms have been proposed to explain the benefit that $\mathrm{CrM}$ has on resistance traininginduced adaptations, including larger lean body mass [18], increased protein expression and synthesis [19], changes in myogenic transcription factors [20], and elevated mitotic activity of satellite cells [21]. Above all, the most likely benefit appears to be due to improved performance during resistance training sessions by increasing intra-muscular phosphocreatine stores, thereby allowing a greater work capacity and thus training stimuli for enhanced chronic training adaptation $[22,23]$. Recent evidence also suggests the potential for CrM supplementation to attenuate muscle damage markers as an acute response to exercise [24]. The mechanical disruption of sarcomeres is considered as the initial event of muscular injury [1]. This primary muscle damage is followed by inflammatory and oxidative stress responses, referred to as the secondary muscle damage response, which increases vascular permeability, oedema, and leukocyte infiltration, resulting in further muscle damage, thus compromising the recovery of muscle structure and function [1]. Interestingly, $\mathrm{CrM}$ has been reported to acutely reduce biomarkers of oxidative stress following strenuous exercise in rats [25], with in vitro data indicating that $\mathrm{CrM}$ exhibits anti-inflammatory effects in endothelial cells [26]. Although these findings may not be directly translatable to humans, they suggest that CrM possesses both anti-inflammatory and antioxidant properties, which would attenuate the secondary muscle damage response.

Several studies have reported reductions in CK, DOMS, inflammation, and oxidative stress, with a concomitant increase in muscle strength following a single bout of strenuous exercise in individuals supplemented with CrM compared with those receiving placebo [27-29] for 24-48 h postexercise. Ingestion of CrM may enhance recovery following strenuous exercise that causes EIMD. In fact, Claudino et al. [30] reported that the level of decrement in lower-body power output was reduced with the ingestion of $\mathrm{CrM}$ when compared with placebo during the pre-season in elite soccer players, thus demonstrating the potential benefit of $\mathrm{CrM}$ to minimise non-functional overreaching. However, when examining the acute responses of the last training session following several weeks of resistance training, studies also reported greater levels of EIMD markers for the CrM group when compared with the placebo group, up to $48 \mathrm{~h}$ post-exercise, despite greater increases in muscle strength in the CrM group [31-33]. Therefore, it would appear that CrM exhibits a paradoxical effect on the EIMD response, whereby this supplement minimises the level of EIMD following a single bout of unfamiliar exercises, although this is reversed if supplemented for several weeks as part of a training programme.

It has been postulated that the increased work capacity typically observed with CrM supplementation may augment the rate of progression of training intensity or volume, resulting 
in superior long-term training adaptation [33]. However, this accelerated progression in training variables may also increase the level of acute physiological stresses [32]. In addition, the ergogenic effects of CrM for recovery vary between 1 and 3 days after strenuous exercises between studies and type of outcome measures, demonstrating the importance of capturing post-exercise stress responses over several days. Furthermore, other studies have also shown no benefit of CrM supplementation on outcome measures associated with EIMD [34-38]. Thus, the effect of CrM on EIMD markers appears conflicting to date, possibly because of distinct methodologies between previous studies, such as the supplementation methods of $\mathrm{CrM}$, training background of participants, the type of muscledamaging protocol, and EIMD outcome measures.

Therefore, a systematic exploration that addresses the methodological discrepancies in these previous studies may clarify the acute and chronic effectiveness of CrM supplementation to minimise EIMD symptoms in different settings. Of note, a systematic review and meta-analysis was conducted recently on the ergogenic effects of $\mathrm{CrM}$ on muscle damage markers [24], with findings indicating that $\mathrm{CrM}$ may reduce $\mathrm{CK}$ at $48 \mathrm{~h}$ post-exercise, although inconclusive results were reported for muscle strength, muscle soreness, and joint range of motion. Although these findings provide important evidence on the effects of CrM based on pooled data from several studies, the chronic effects of training and CrM supplementation on muscle damage markers have not yet been examined. Furthermore, inflammatory and oxidative stress markers during periods of EIMD are indicative of the secondary muscle damage response [1], although these biomarkers were not assessed in the systematic review by Northeast and Clifford [24]. Expanding on these outcome measures may demonstrate further impact of $\mathrm{CrM}$ as an ergogenic aid and shed light on the potential mechanisms underpinning greater training adaptation. Therefore, the purpose of the current systematic review and meta-analysis was twofold: to investigate the effect of CrM supplementation on various biomarkers (indirect muscle damage, inflammation, and oxidative stress) and muscular strength measures following a single bout of strenuous activities and to examine these outcome measures following the last bout of several weeks of training.

\section{Methodology}

This systematic review has been registered with PROSPERO (registration number: CRD42020207421) and was conducted following the PRISMA (Preferred Reporting Items for Systematic Reviews and Meta-Analyses) guidelines [39]. The PICO (population, intervention/exposure, comparison and outcome) approach was used to construct the inclusion criteria, with the following inclusion and exclusion criteria:
- Population: healthy male and female humans.

- Intervention: ingestion of oral CrM supplements.

- Exposure: exercises employed to cause EIMD, such as isokinetic eccentric contractions, resistance training, plyometrics, and running.

- Comparison: the outcome measures were compared between the CrM and placebo groups at 24-36 and 48-90 $\mathrm{h}$ after the muscle-damaging exercises.

- Outcome: the outcome measures included blood biomarkers of indirect muscle damage (i.e., CK, myoglobin and lactate dehydrogenase $[\mathrm{LDH}]$ ) and subjective measures of muscle soreness (i.e., visual analogue scale) and muscular performance (i.e., isometric or isokinetic torque, vertical jump and maximum strength).

The exclusion criteria were as follows: (1) studies conducted in animals; (2) studies where CrM supplements were used to induce chronic adaptations, such as assessment of strength development after 6 weeks of resistance training with CrM supplementation without measurement of indirect muscle damage, muscle soreness, and acute responses to muscular performance; (3) studies with outcome measures reported immediately after $(<24 \mathrm{~h})$ or $>90 \mathrm{~h}$ after the muscle-damaging exercises; (4) studies published in nonEnglish languages; (5) study results published as conference proceedings, reviews, and case reports.

\subsection{Search Strategy}

A literature search was performed from 27 August 2021 using the PubMed, Scopus, SPORTDiscus, CINAHL, and Web of Science databases. Four strings of medical subject heading terms were employed for the PubMed search: (1) adult or young adult; (2) supplements (dietary supplements); (3) indirect muscle damage markers (creatine kinase; muscle, skeletal; 1-lactate dehydrogenase; pain/drug therapy; pain/aetiology; muscle fatigue/drug effects; muscle fatigue/ physiology; myalgia/drug therapy; myalgia/prevention and control); and (4) exercise (exercise test; exercise tolerance/ physiology; physical endurance/physiology; physical exertion/physiology; physical endurance/drug effects; exercise; resistance training; muscle contraction; running/physiology). A free-text search was conducted for CINAHL, Scopus, SPORTDiscus, and Web of Science with the following strings: (muscle damage or creatine kinase or lactate dehydrogenase or myoglobin or soreness) and (creatine monohydrate or creatine supplementation). These free-text search strings were also used for PubMed but only for an 18-month time limit to capture publications that were still 'in press'. For the supplementary search, screening was also conducted in Google Scholar and the reference lists of all included studies. 


\subsection{Selection Process}

Two experienced exercise scientists (JDC and AKR) completed the screening process. First, the abstracts (with duplicates removed) from all databases were screened using the criteria of either 'yes' (meeting the inclusion criteria), 'maybe' (possibly meeting the inclusion criteria), or 'no' (not meeting the inclusion criteria). Any inter-rater discrepancy was discussed with another exercise scientist (KD) until a consensus was reached. Once abstract screening was completed, the full-text articles were further screened based on the inclusion criteria.

\subsection{Data Extraction, Assessment of Quality, and Risk of Bias}

The descriptive information regarding study aims, participant characteristics (e.g., age, height, body mass, body mass index [BMI], training background), research design (i.e., cross-over randomised or randomised controlled placebo), the type of biomarker for muscle damage (e.g., CK, myoglobin, and LDH) and inflammation (e.g., ILs, C-reactive protein $[\mathrm{CRP}]$, tumour necrosis factor $[\mathrm{TNF}]-\alpha)$, the type of muscle performance measures (e.g., isokinetic/isometric knee extension), and the post-exercise time points (i.e., either 24 or $48 \mathrm{~h}$ post-exercise) was entered into a Microsoft Excel spreadsheet. All continuous outcome measures were extracted from each study as mean \pm standard deviation (SD) to create forest plots and compare results between the $\mathrm{CrM}$ and placebo groups at the selected post-exercise time points. We used the PEDro rating scale as the critical appraisal tool; this originally consisted of an 11-point scoring system to assess the quality of randomised controlled trials in the Physiotherapy Evidence Database [40]. However, we modified this critical appraisal tool by incorporating four additional criteria to align the study design specifically in supplemental research and EIMD [13, 41], including (1) resistance training background of participants; (2) bioavailability of $\mathrm{CrM}$; (3) reporting of active ingredients according to the manufacturer's nutritional label; and (4) supplemental and medicinal habits of participants. As with the original PEDro criteria, the first three additional criteria were scored either as 1 (meeting the item) or 0 (not meeting the item). However, the final additional criterion was scored 2 if participants were prevented from taking CrM supplementation before the study and anti-inflammatory medication during the study, 1 if participants were prevented from taking CrM supplementation before the study or anti-inflammatory medication during the study, or 0 if the criterion was not met. Therefore, a maximum score of 16 was achievable with this modified PEDro scale, and the classification of the quality of the ratings was as follows: excellent (score 14-16); good (11-13); fair (8-10), and poor $(<7)$ [13]. The second author (AKR) rated each study using this modified PEDro scale. To ascertain potential publication bias of the pooled data from each study, funnel plots were created using meta-analytical software (RevMan, version 5.3, Copenhagen: The Nordic Cochrane Centre, 2014). Egger's test was also conducted using a linear regression of the normalised effect estimates against their inverse variance to calculate the y-intercept and associated p-values [42] using a statistical software package (SPSS, v24; Chicago, IL, USA), with a $p$-value $<0.05$ implicating a publication bias. However, Egger's test was only carried out for outcome measures that consisted of at least ten studies [42] at each time point (i.e., T24 and/or T48), given that the power to detect bias for this method is low with a smaller number of studies. Participant selection bias was controlled by selecting studies of all healthy adults, irrespective of sex and training background.

\subsection{Statistical Analysis}

The means and SDs of the outcome measures were extracted from each study and pooled to meta-analytically compare data between the $\mathrm{CrM}$ and placebo groups at 24 and $48 \mathrm{~h}$ following the muscle-damaging protocol. If the measure of dispersion was reported as either a 95\% confidence interval or a standard error, we converted it to an SD before imputing data into the software (RevMan), based on previous recommendations [42]. Furthermore, if a study reported multiple outcome measures that measured the same phenomena (e.g., CK, myoglobin, and LDH for indirect muscle damage markers), we calculated the average to report on a singular effect estimate [43]. Once all effect estimates were combined into the statistical software, forest plots were generated using the random effects model given that the methodological design, such as participant background, muscle-damaging protocols, and biomarkers, varied between studies. Furthermore, inter-study heterogeneity was reported, with $\mathrm{I}^{2}$ values of $25 \%, 50 \%$, and $75 \%$ interpreted as low, moderate, and high, respectively. The SMDs between the CrM and placebo groups were also derived from the forest plot at 24 and $48 \mathrm{~h}$ following the muscle-damaging exercise. The SMD values of $0.2,0.5$, and 0.8 were classified as small, moderate, and large, respectively [44]. The $Z$-value was also calculated to report on the effect of the pooled data between the CrM and placebo groups, with $p$-values corresponding to the level of statistical significance. We also conducted a sensitivity analysis to determine the impact of potential outliers in the forest plot. 


\section{Results}

\subsection{Systematic Literature Search}

After removing 242 duplicate abstracts, 1865 abstracts from the five databases were screened according to the inclusion criteria (Fig. 1). After abstract screening, 1824 abstracts were excluded, 41 full-text articles were further screened, and the remaining 23 articles were included in this review. All studies employed a parallel design, consisting of a group that ingested $\mathrm{CrM}$ and a group that ingested a placebo alternative.

\subsection{Participants}

From the included studies, data were extracted from 469 participants, of which 240 and 229 participants were in the CrM and placebo groups, respectively. The mean age, height, and body mass and percentage of females was $23.9 \pm 10.4$ years, $178 \pm 5 \mathrm{~cm}, 76.9 \pm 7.6 \mathrm{~kg}$, and $10.4 \%$, respectively, for the CrM group and $23.7 \pm 8.5$ years, $177 \pm 5 \mathrm{~cm}, 77.0 \pm 6.6 \mathrm{~kg}$, and $10.0 \%$, respectively, for the placebo group. As such, the physical characteristics were evenly distributed between the $\mathrm{CrM}$ and placebo groups (Table 1). Furthermore, there were no significant differences in the outcome measures between the $\mathrm{CrM}$ and placebo groups $(p>0.05)$ reported at baseline or before the muscle-damaging exercise for all but one study, suggesting that the outcome measures for both groups were relatively homogenous.

\subsection{Methodological Descriptions}

The most common muscle-damaging exercises were in the order of eccentric/concentric resistance exercises (11 studies), eccentric contractions (four studies), middle-tolong distance running (four studies), graded exercise test (two studies), downhill running (one study), and vertical jump test (one study) (Table 2). The most frequently reported biomarker for indirect muscle damage was CK (16 studies), followed by LDH (ten studies). Several types of inflammatory biomarkers were reported, including IL-6 (two studies), TNF $\alpha$ (two studies), CRP (one study), interferon- $\alpha$ (one study), and IL-1 $\beta$ (one study). Similarly,
Fig. 1 Schematic of the PRISMA flowchart

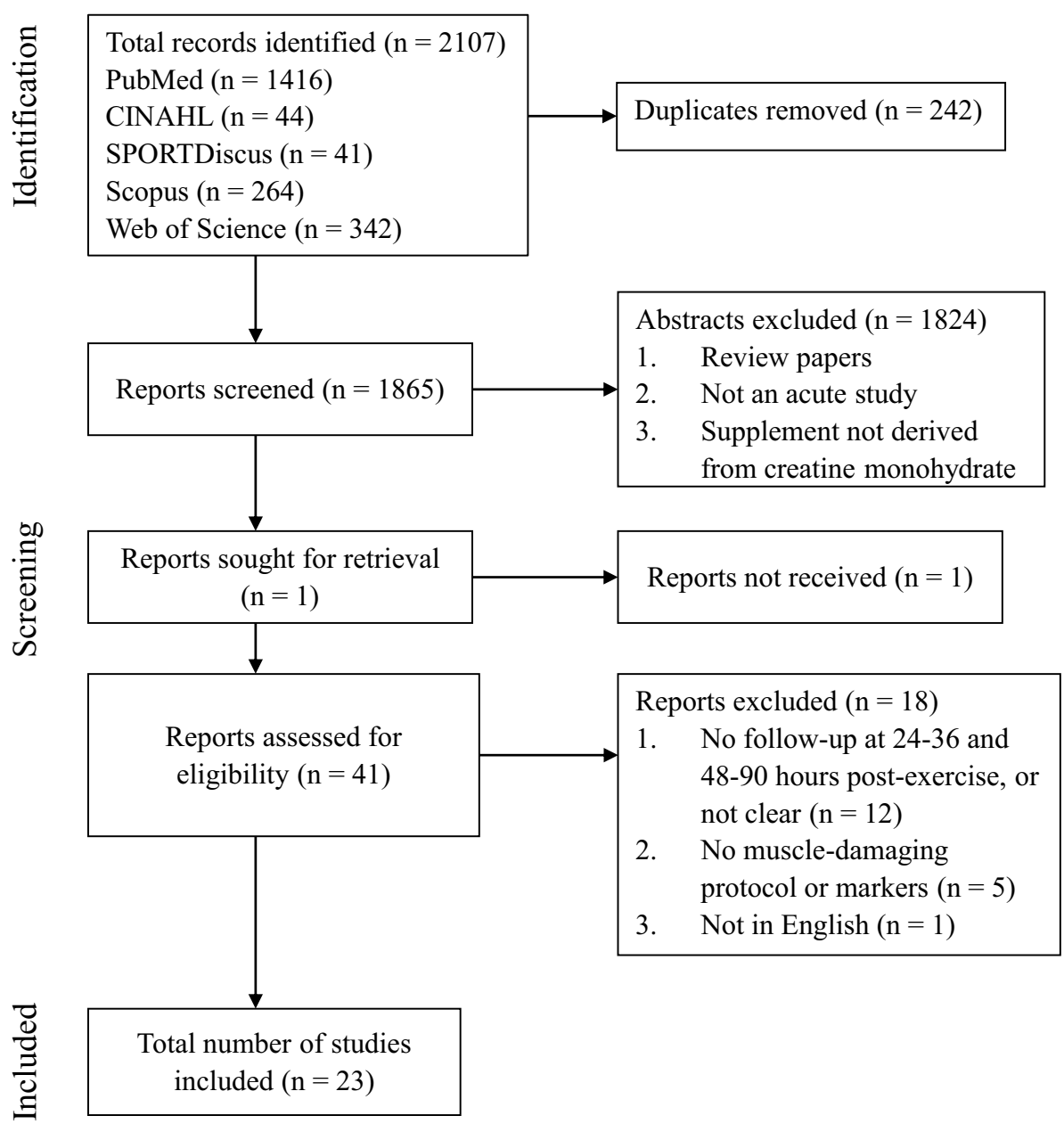




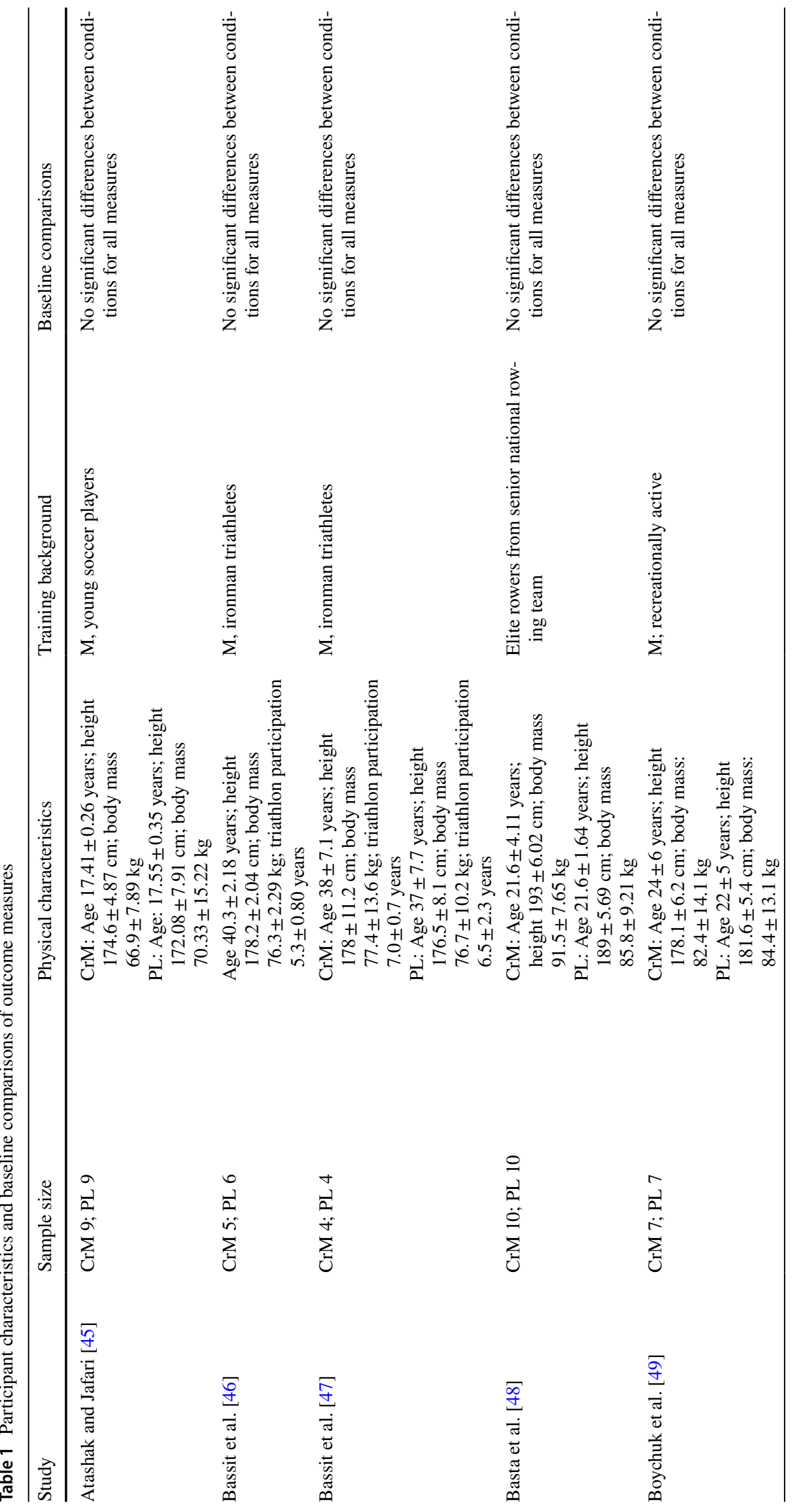




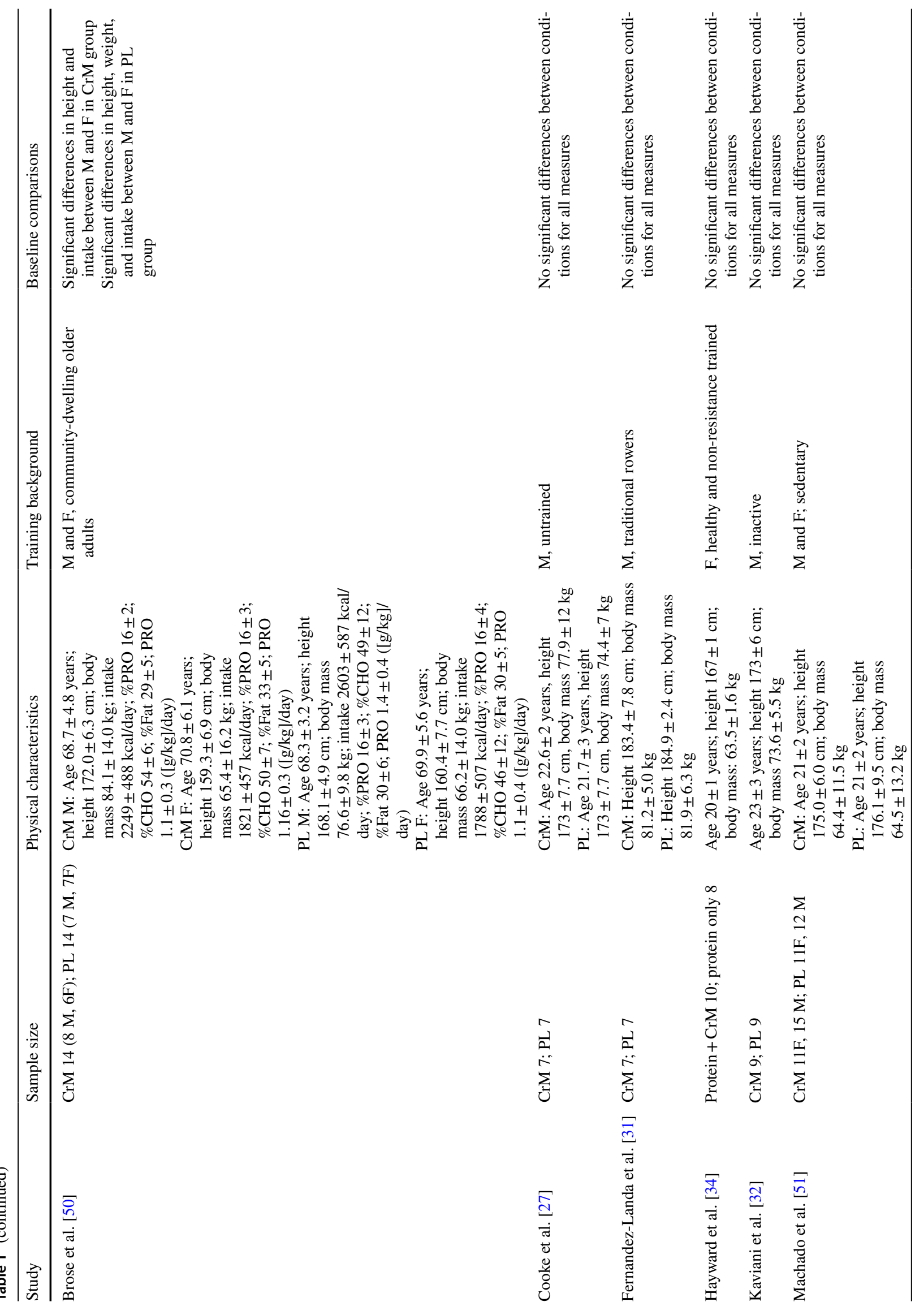




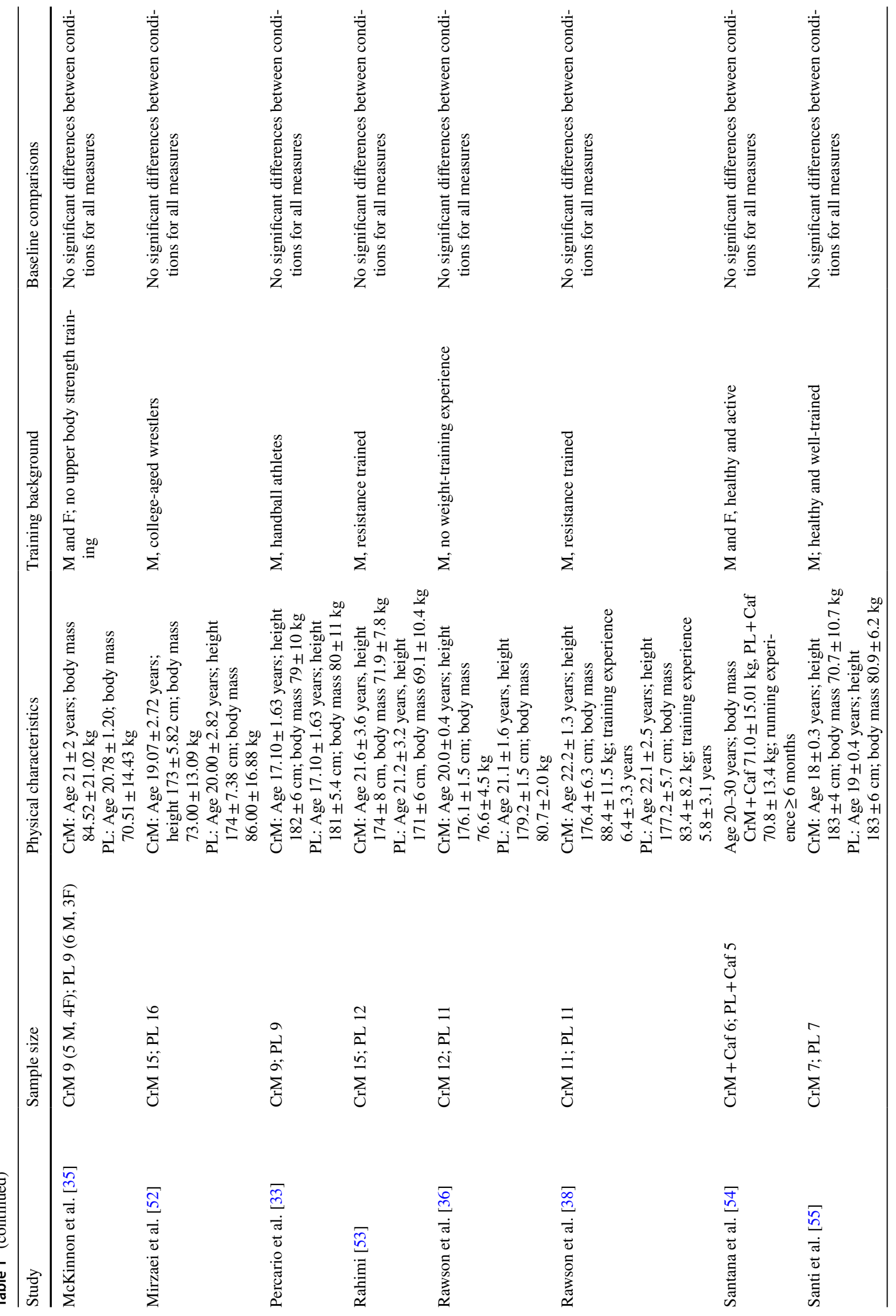




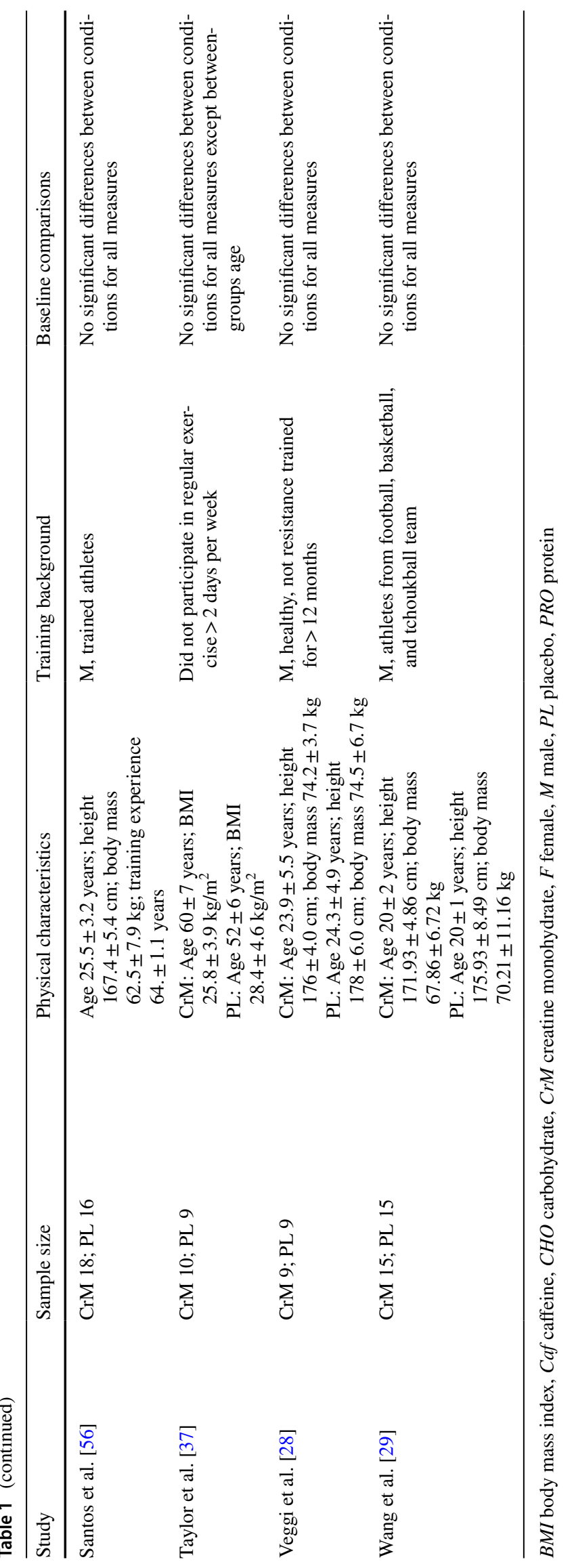




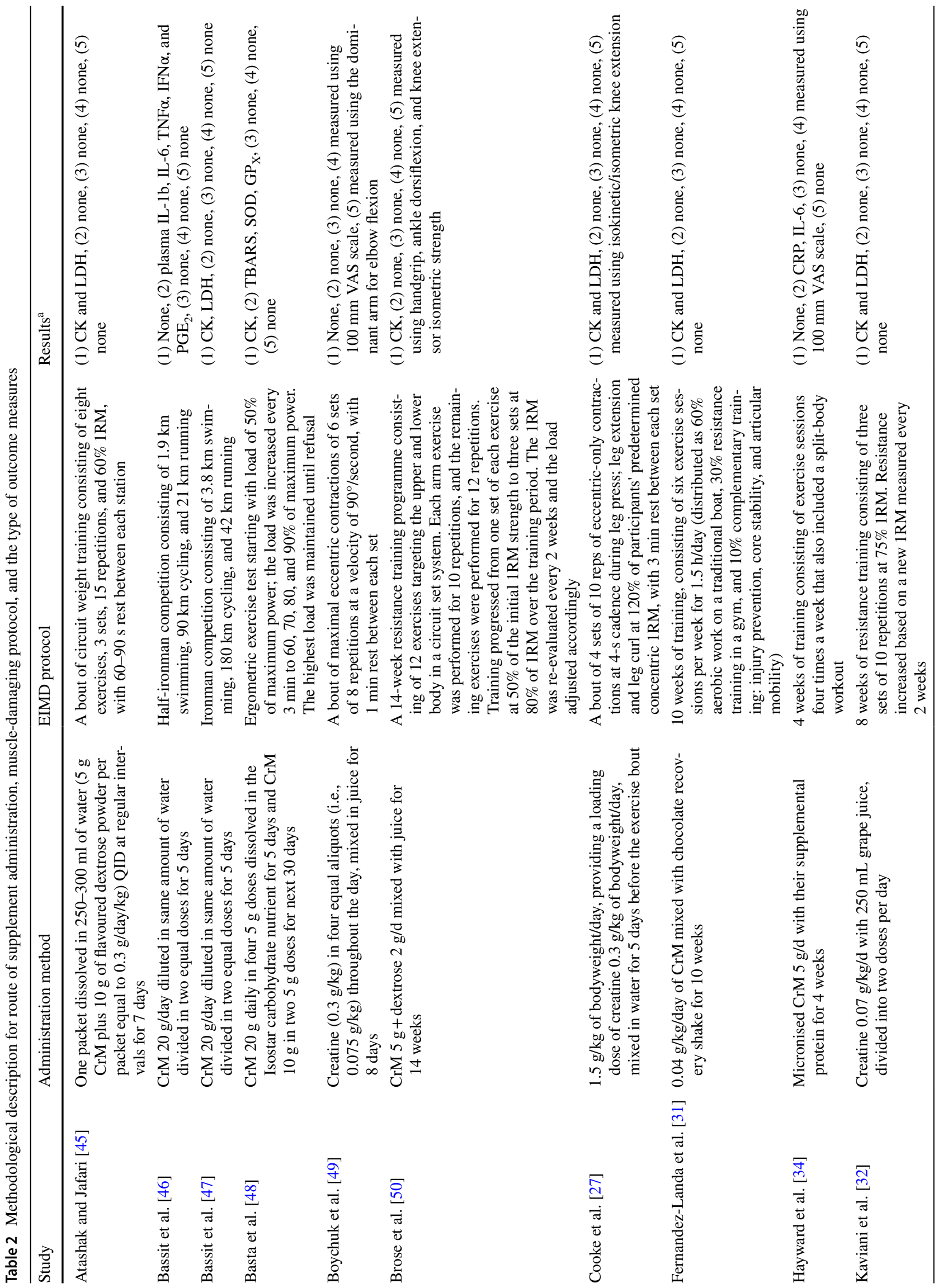




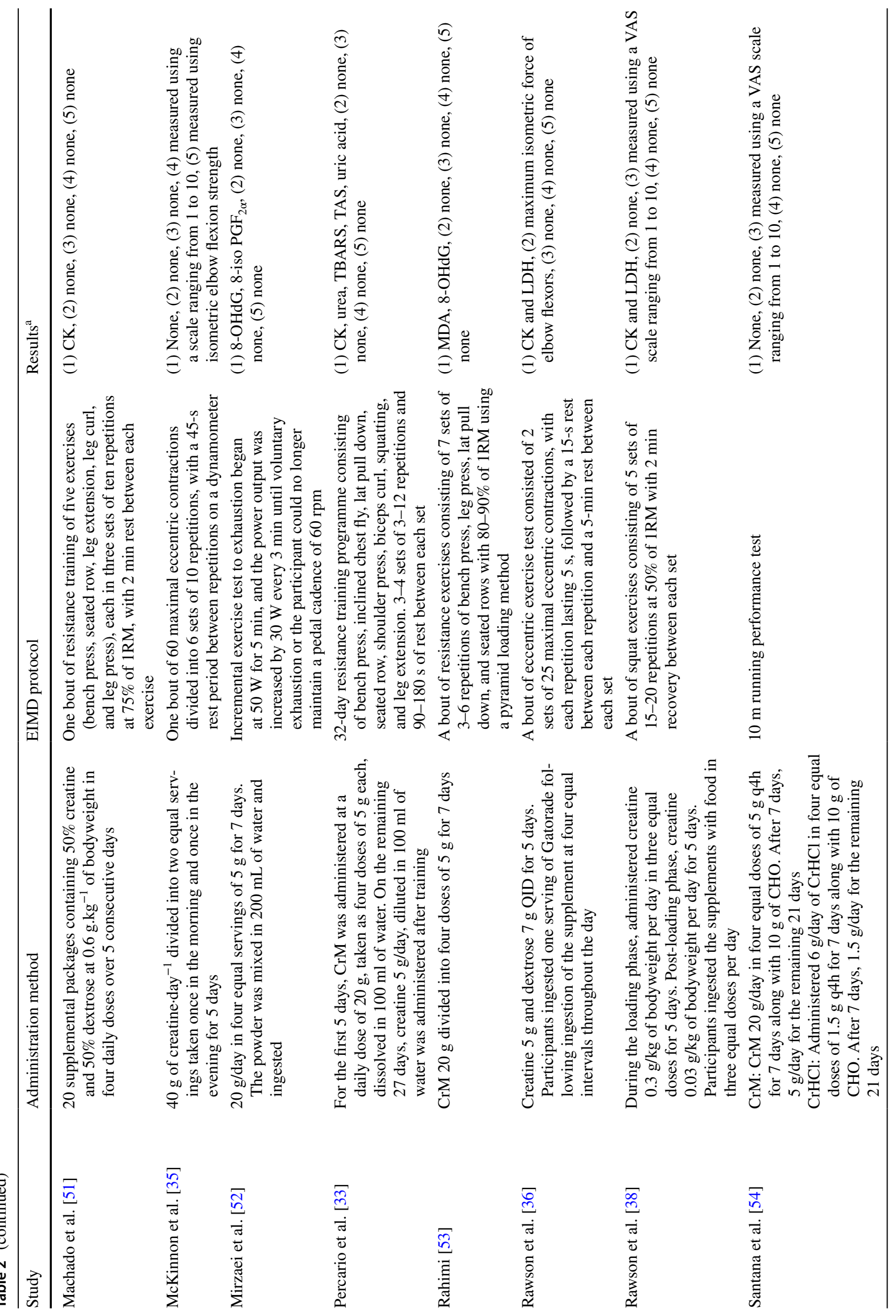




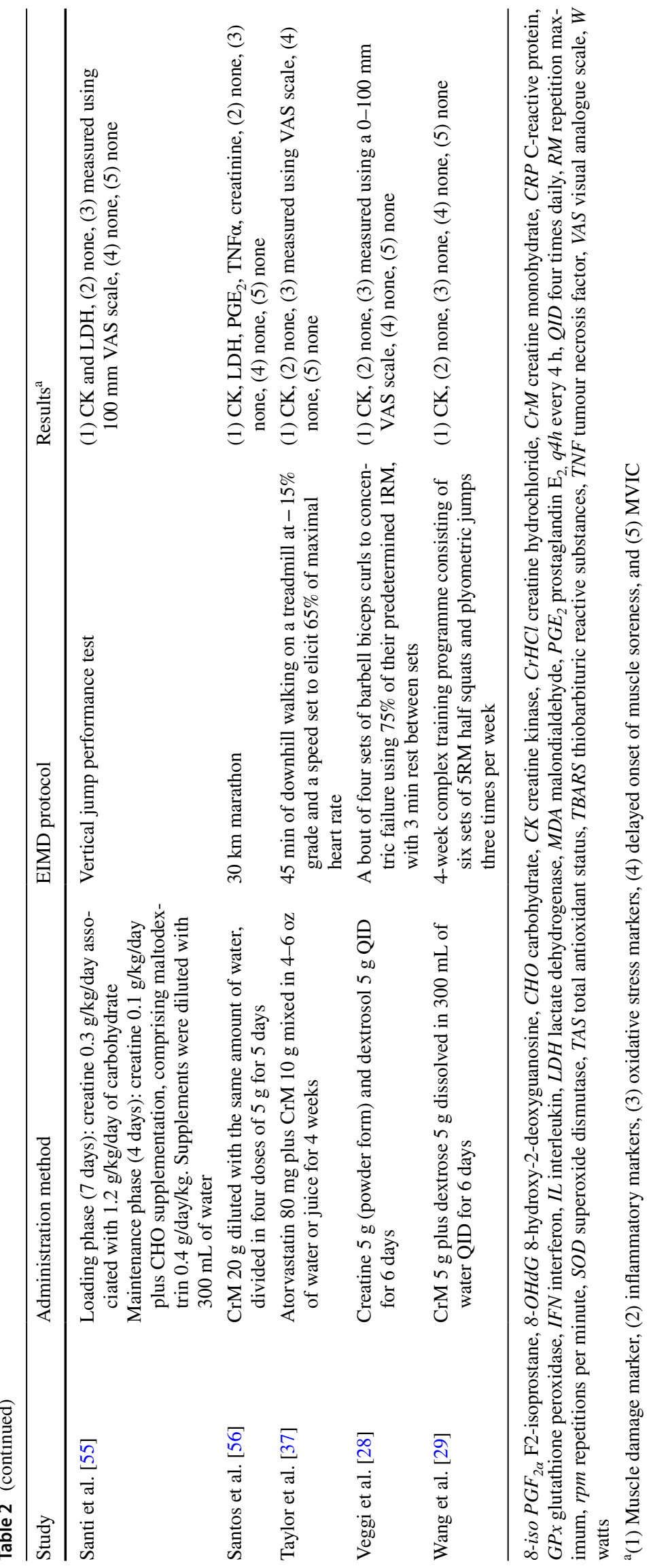


Table 3 PEDro ratings of all included studies

\begin{tabular}{|c|c|c|c|c|c|c|c|c|c|c|c|c|c|c|c|c|c|}
\hline Study & 1 & 2 & 3 & 4 & 5 & 6 & 7 & 8 & 9 & 10 & 11 & 12 & 13 & 14 & 15 & Ratings & Quality \\
\hline Atashak and Jafari [45] & 1 & 1 & 1 & 0 & 1 & 1 & 0 & 1 & 1 & 0 & 1 & 1 & 1 & 1 & 1 & $12 / 16$ & Good \\
\hline Bassit et al. [46] & 1 & 1 & 1 & 0 & 1 & 1 & 1 & 1 & 1 & 0 & 1 & 1 & 1 & 1 & 1 & $13 / 16$ & Excellent \\
\hline Bassit et al. [47] & 1 & 1 & 1 & 0 & 1 & 1 & 1 & 1 & 1 & 0 & 1 & 1 & 1 & 1 & 1 & $13 / 16$ & Excellent \\
\hline Basta et al. [48] & 1 & 0 & 1 & 0 & 1 & 0 & 0 & 0 & 0 & 0 & 1 & 1 & 1 & 1 & 1 & $8 / 16$ & Fair \\
\hline Boychuk et al. [49] & 1 & 2 & 1 & 0 & 1 & 1 & 1 & 1 & 1 & 0 & 1 & 1 & 1 & 1 & 1 & $14 / 16$ & Good \\
\hline Brose et al. [50] & 1 & 0 & 1 & 0 & 0 & 0 & 0 & 1 & 1 & 1 & 1 & 1 & 1 & 1 & 1 & $10 / 16$ & Fair \\
\hline Cooke et al. [26] & 1 & 2 & 1 & 0 & 1 & 1 & 1 & 1 & 1 & 0 & 1 & 1 & 1 & 1 & 1 & $14 / 16$ & Good \\
\hline Fernandez-Landa et al. [30] & 1 & 1 & 1 & 1 & 1 & 1 & 0 & 1 & 1 & 0 & 1 & 1 & 1 & 1 & 1 & $13 / 16$ & Good \\
\hline Hayward et al. [33] & 1 & 1 & 1 & 0 & 1 & 1 & 1 & 0 & 0 & 0 & 1 & 1 & 1 & 1 & 1 & $11 / 16$ & Good \\
\hline Kaviani et al. [31] & 1 & 1 & 1 & 0 & 1 & 1 & 1 & 1 & 1 & 0 & 0 & 1 & 1 & 1 & 1 & $12 / 16$ & Good \\
\hline Machado et al. [51] & 1 & 1 & 1 & 1 & 1 & 0 & 0 & 1 & 1 & 0 & 1 & 1 & 1 & 1 & 1 & $12 / 16$ & Good \\
\hline McKinnon et al. [34] & 1 & 1 & 1 & 1 & 1 & 0 & 1 & 1 & 1 & 0 & 1 & 1 & 1 & 1 & 1 & $13 / 16$ & Good \\
\hline Mirzaei et al. [52] & 1 & 1 & 1 & 0 & 1 & 1 & 0 & 1 & 1 & 0 & 1 & 1 & 1 & 1 & 1 & $12 / 16$ & Good \\
\hline Percario et al. [32] & 1 & 1 & 1 & 0 & 1 & 1 & 0 & 1 & 1 & 1 & 1 & 1 & 1 & 1 & 1 & $13 / 16$ & Excellent \\
\hline Rahimi [53] & 1 & 1 & 1 & 0 & 1 & 1 & 1 & 1 & 1 & 0 & 0 & 1 & 1 & 1 & 1 & $12 / 16$ & Good \\
\hline Rawson et al. [35] & 1 & 0 & 1 & 0 & 1 & 1 & 1 & 1 & 1 & 0 & 1 & 1 & 1 & 1 & 1 & $12 / 16$ & Good \\
\hline Rawson et al. [37] & 1 & 1 & 1 & 0 & 1 & 1 & 1 & 1 & 1 & 0 & 1 & 1 & 1 & 1 & 1 & $13 / 16$ & Good \\
\hline Santana et al. [54] & 1 & 1 & 1 & 0 & 1 & 0 & 1 & 1 & 1 & 0 & 1 & 1 & 1 & 1 & 1 & $12 / 16$ & Good \\
\hline Santi et al. [55] & 1 & 1 & 1 & 0 & 1 & 1 & 0 & 1 & 1 & 1 & 1 & 1 & 1 & 1 & 1 & $13 / 16$ & Good \\
\hline Santos et al. [56] & 1 & 2 & 1 & 0 & 1 & 1 & 1 & 1 & 1 & 1 & 1 & 1 & 1 & 1 & 1 & $15 / 16$ & Excellent \\
\hline Taylor et al. [36] & 1 & 2 & 1 & 0 & 0 & 0 & 1 & 1 & 1 & 0 & 1 & 1 & 1 & 1 & 1 & $12 / 16$ & Good \\
\hline Veggiv [27] & 1 & 1 & 1 & 0 & 1 & 1 & 1 & 0 & 0 & 0 & 1 & 1 & 1 & 1 & 1 & $11 / 16$ & Good \\
\hline Wang et al. [28] & 1 & 2 & 1 & 0 & 1 & 1 & 0 & 1 & 1 & 0 & 1 & 1 & 1 & 1 & 1 & $13 / 16$ & Good \\
\hline
\end{tabular}

various oxidative stress markers were reported, including thiobarbituric acid reactive substances (TBARS; two studies), 8-Oxo-2'-deoxyguanosine (8-OHdG; two studies), glutamic oxaloacetic transaminase (one study), glutathione peroxidase (one study), malondialdehyde (one study), and 8-iso-prostaglandin F2 $\alpha$ (one study). With respect to the measures of DOMS, the most common forms of visual analogue scale (VAS) consisted of $0-100 \mathrm{~mm}$ (four studies), followed by VAS scales of 1-10 (three studies), and 0-25 cm (one study). The most frequent muscle performance protocol consisted of isometric contractions (four studies), followed by isokinetic contraction (one study).

\subsection{Methodological Quality}

The scores from the PEDro scale indicated a range from fair to excellent quality (Table 3 ). The following PEDro items were addressed by all studies: baseline values were standardised between the $\mathrm{CrM}$ and placebo groups; outcome measures were reported for more than $85 \%$ of the participants; data were treated similarly irrespective of group allocation; all participants received either CrM or placebo; and appropriate statistical analyses were conducted to compare data between groups. Most studies reported the measure of dispersion (either SD, standard error, or confidence intervals); participants were randomly allocated into CrM and placebo groups; participants were instructed to refrain from pain medication/supplements and CrM supplementation before and during the study; a double-blind method was employed; and participants were homogenous. The fewest PEDro items addressed included specificity of resistance training background; concealment of allocation; and reporting of the bioavailability of the CrM supplement.

\subsection{Quantitative Analyses}

With respect to indirect muscle damage markers (i.e., CK, $\mathrm{LDH}$, and myoglobin), no significant differences were found between the $\mathrm{CrM}$ and placebo groups for the training response $(p=0.45)$, with small effect sizes (SMD -0.23 ; $I^{2}=78 \%$ ) at $24-36 \mathrm{~h}$ post-exercise (Fig. 2a). However, the indirect muscle damage markers at $48-90 \mathrm{~h}$ post-exercise were significantly greater in the placebo group for the acute training response $(p=0.03)$, with a large effect size (SMD $-1.09 ; I^{2}=83 \%$; Fig. $2 b$ ). Furthermore, indirect muscle damage markers were significantly greater in the CrM group for the chronic training response at $24 \mathrm{~h}(p=0.04)$, with a large effect size (SMD 0.95; $I^{2}=67 \%$; Fig. 2a). Although not significant $(p=0.06)$, the muscle damage 
(a)

\begin{tabular}{|c|c|c|}
\hline \multirow{2}{*}{ Study or Subgroup } & \multicolumn{2}{|r|}{ Std. Mean Difference } \\
\hline & Weight & IV, Random, $95 \% \mathrm{Cl}$ \\
\hline \multicolumn{3}{|l|}{ 1.1.1 Acute training response } \\
\hline Atashak et al 2012 [45] & $8.9 \%$ & $1.28[0.24,2.31]$ \\
\hline Bassit et al 2010 [47] & $6.6 \%$ & $-1.14[-2.73,0.45]$ \\
\hline Cooke et al 2009 [26] & $7.1 \%$ & $-2.27[-3.72,-0.83]$ \\
\hline Wachado et al 2009 (Men) [51] & $10.0 \%$ & $0.82[0.02,1.61]$ \\
\hline Wachado et al 2009 (Women) [51] & $9.5 \%$ & $-1.00[-1.89,-0.10]$ \\
\hline Rawson et al 2001 [35] & $9.8 \%$ & $0.58[-0.26,1.42]$ \\
\hline Rawson et al 2007 [37] & $9.7 \%$ & $-0.45[-1.30,0.40]$ \\
\hline Santi et al 2020 [55] & $8.6 \%$ & $0.71[-0.38,1.80]$ \\
\hline Santos et al 2004 [56] & $10.1 \%$ & $-1.36[-2.12,-0.61]$ \\
\hline Taylor et al 2018 [36] & $9.4 \%$ & $0.45[-0.47,1.36]$ \\
\hline Wang & $10.2 \%$ & \\
\hline Subtotal $(95 \% \mathrm{Cl})$ & $100.0 \%$ & $-0.23[-0.84,0.37]$ \\
\hline \multicolumn{3}{|c|}{$\begin{array}{l}\text { Heterogeneity: } \mathrm{Tau}^{2}=0.7 \mathrm{~g} ; \mathrm{Chi}^{2}=45.53, \mathrm{df}=10(\mathrm{P}=0.00001) ; \mathrm{I}^{2}=78 \\
\text { Test for owerall effect: } Z=0.75(\mathrm{P}=0.45)\end{array}$} \\
\hline \multicolumn{3}{|l|}{ 1.1.2 Chronic Training response } \\
\hline Basta et al 2006 [48] & $27.9 \%$ & $0.37[-0.52,1.2$ \\
\hline Fernandez-Landa et al 2020 [30] & $25.0 \%$ & $0.42[-0.64,1.49]$ \\
\hline Kaviani et al 2019 [31] & $20.5 \%$ & $2.72[1.35,4.08]$ \\
\hline $\begin{array}{l}\text { Percario et al } 2012 \text { [32] } \\
\text { Subtotal } 95 \% \mathrm{Cl}\end{array}$ & $26.7 \%$ & $0.71[-0.25,1.67]$ \\
\hline Subtotal $(95 \% \mathrm{Cl})$ & $100.0 \%$ & \\
\hline \multicolumn{3}{|c|}{$\begin{array}{l}\text { Heterogeneity: } \operatorname{Tau}^{2}=0.57 ; \mathrm{Chi}^{2}=9.00, \mathrm{df}=3(\mathrm{P}=0.03) ; \mathrm{I}^{2}=67 \% \\
\text { Test for owerall effect: } \mathrm{Z}=2.06(\mathrm{P}=0.04)\end{array}$} \\
\hline
\end{tabular}

Std. Mean Difference IV, Random, $95 \% \mathrm{Cl}$

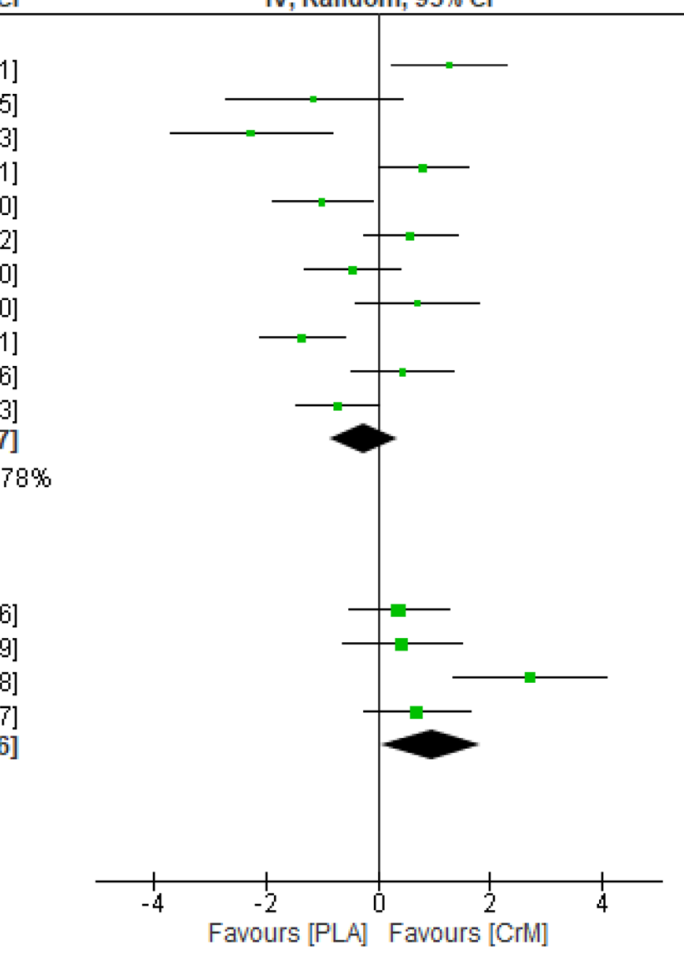

(b)

\begin{tabular}{|c|c|c|}
\hline Study or Subgroup & Weight & IV, Random, $95 \% \mathrm{Cl}$ \\
\hline \multicolumn{3}{|l|}{ 1.2.1 Acute training response } \\
\hline Bassit et al 2010 [47] & $9.5 \%$ & $-2.19[-4.24,-0.14]$ \\
\hline Cooke et al 2009 [26] & $8.2 \%$ & $-4.88[-7.26,-2.51]$ \\
\hline Rawson et al 2001 [35] & $14.8 \%$ & $-0.25[-1.08,0.57]$ \\
\hline Rawson et al 2007 [37] & 14. & $87,-0.08$ \\
\hline Banti & $13.8 \%$ & \\
\hline Taylo & 14. & \\
\hline Veg & $9.6 \%$ & -4 \\
\hline Wan & 15. & \\
\hline Subtotal $(95 \% \mathrm{C}$ & $100.0 \%$ & $-1.09[-2$ \\
\hline \multicolumn{3}{|c|}{$\begin{array}{l}\text { Heterogeneity: } \operatorname{Tau}^{2}=1.46 ; \mathrm{Ch}^{2}=40.84, \mathrm{df}=7(\mathrm{P}=0.00001) ;\left.\right|^{2}= \\
\text { Test for overall effect: } \mathrm{Z}=2.22(\mathrm{P}=0.03)\end{array}$} \\
\hline \multicolumn{3}{|l|}{ 1.2.2 Chronic training response } \\
\hline Brose et al 2003 (Men) [50] & 35. & 0.3 \\
\hline men $[50]$ & 33.3 & $0.85[-0$. \\
\hline Kaviani et al 2019 [31] & $31.1 \%$ & \\
\hline Subtotal $(95 \% \mathrm{Cl})$ & $100.0 \%$ & $1.24[-0.05,2.5$ \\
\hline
\end{tabular}

Study or Subgroup

$8.2 \%$

$-4.89[-7.26,-2.51]$

$0.25[-1.08,0.57]$

$-0.98[-1.87,-0.08]$

$0.35[-0.71,1.40]$

$[-0.27,1.59]$

$-4.41[-6.43,-2.39]$

$-0.14[-0.86,0.57]$

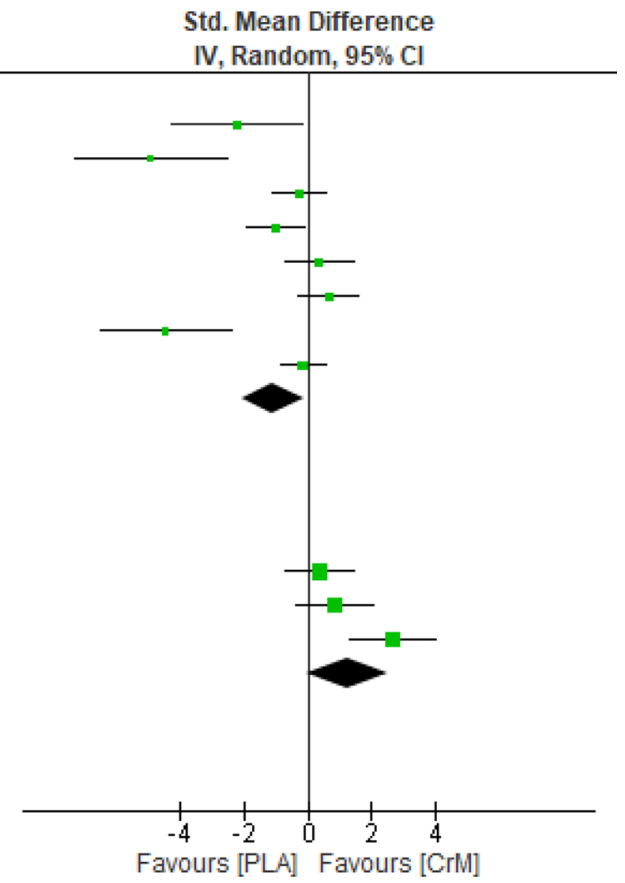

Fig. 2 Forest plot for indirect muscle damage markers at $\mathbf{a} 24$ and b $48 \mathrm{~h}$ after the muscle-damaging protocol. $\mathrm{CrM}$ creatine monohydrate group, $P L A$ placebo group

markers were greater for the CrM group as a chronic training response at $48 \mathrm{~h}$ post-exercise, with a large effect size (SMD $1.24 ; I^{2}=71 \%$; Fig. $2 b$ ). The inflammatory markers appeared larger in the placebo group, with a large effect size for the acute training response at $24-36 \mathrm{~h}$ (SMD -0.91 ; $I^{2}=83 \%$; Fig. 3a) and at $48-90 \mathrm{~h}\left(\mathrm{SMD}-1.79 ; I^{2}=86 \%\right.$; 


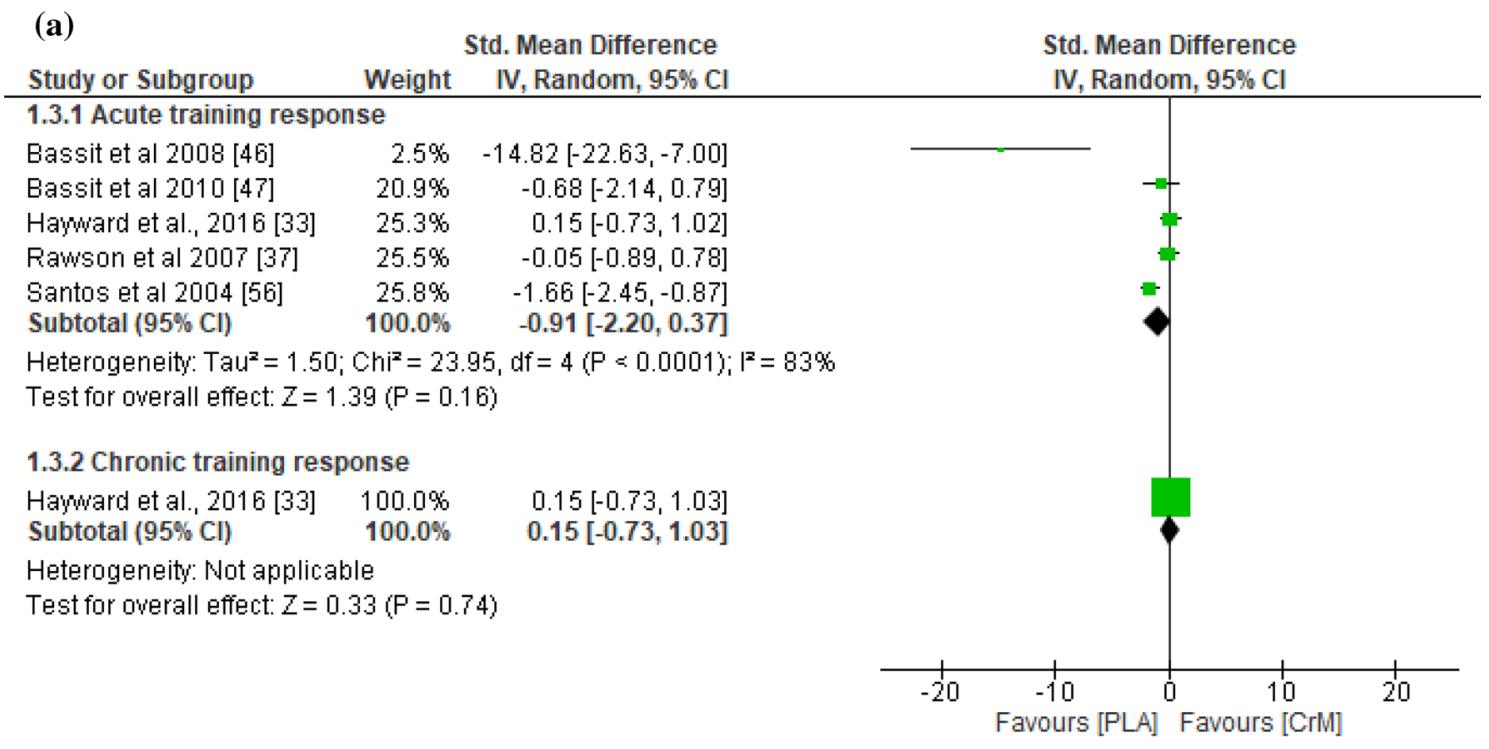

(b)

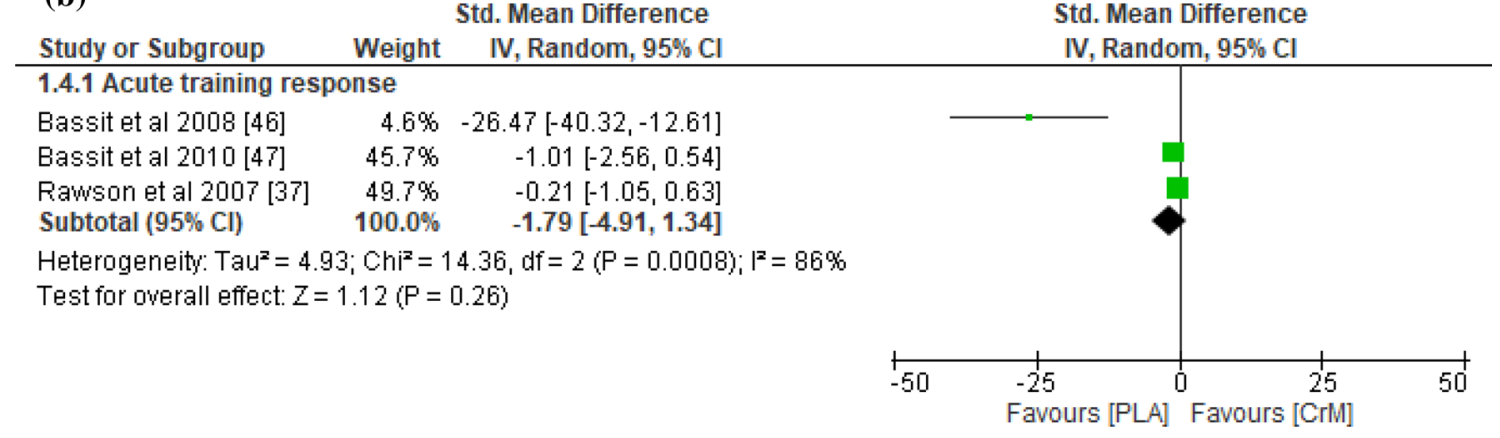

Fig. 3 Forest plot for inflammatory markers at (a) 24 and (b) $48 \mathrm{~h}$ after the muscle-damaging protocol. CrM creatine monohydrate group, $P L A$ placebo group

Fig. 3b) post-exercise, although no significant inter-group differences were evident $(p>0.05)$. Furthermore, no differences were found in inflammatory markers at $24 \mathrm{~h}$ postexercise for the chronic training response $(p=0.74)$, with a small effect size (SMD 0.15). The oxidative stress markers were significantly greater in the placebo group for the acute training response at $24-36 \mathrm{~h}$ post-exercise $(p<0.001)$, with a large effect size (SMD $-1.37 ; I^{2}=0 \%$; Fig. $4 \mathrm{a}$ ), although there were no inter-group differences for the chronic training $(p=0.47)$, with a small effect size (SMD $0.24 ; I^{2}=0 \%$; Fig. 4a). Although there were no inter-group differences in oxidative stress markers for the acute training response at $90 \mathrm{~h}$ post-exercise $(p=0.11)$, a large effect size was found with values greater for the placebo group (SMD - 1.36; Fig. 4b). There were no significant inter-group differences in DOMS for the acute and chronic training responses at 24 and 48 h (Fig. 5a and b, respectively) post-exercise $(p>0.05)$. However, the DOMS measures were greater for the placebo group, with a moderate effect size at $24 \mathrm{~h}$ (SMD - 0.66; $I^{2}=89 \%$; Fig. 5a), but a small effect size at $48 \mathrm{~h}$ (SMD $-0.49 ; I^{2}=77 \%$; Fig. $5 \mathrm{~b}$ ) post-exercise for the acute training response. The DOMS appeared larger for the $\mathrm{CrM}$ group at $24 \mathrm{~h}$ post-exercise for the chronic training response, but with a small effect size (SMD $0.45 ; I^{2}=78 \%$; Fig. 5a). There were no inter-group differences in muscle force measures for the acute training responses at 24 and $48 \mathrm{~h}$ (Fig. 6a and b, respectively) post-exercise $(p>0.05)$, with small effect sizes (SMD -0.48 and $0.29 ; I^{2}=86 \%$ and $57 \%$; respectively).

\subsection{Sensitivity Analysis}

According to the sensitivity analysis, potential outliers postexercise were identified for muscle damage at $24 \mathrm{~h}$ [27] and $48 \mathrm{~h} \mathrm{[28],} \mathrm{inflammatory} \mathrm{markers} \mathrm{at} 24 \mathrm{~h} \mathrm{[46]} \mathrm{and} 48 \mathrm{~h} \mathrm{[46],}$ oxidative stress at $24 \mathrm{~h} \mathrm{[47],} \mathrm{DOMS} \mathrm{at} 24 \mathrm{~h} \mathrm{[38]} \mathrm{and} 48 \mathrm{~h}$ 


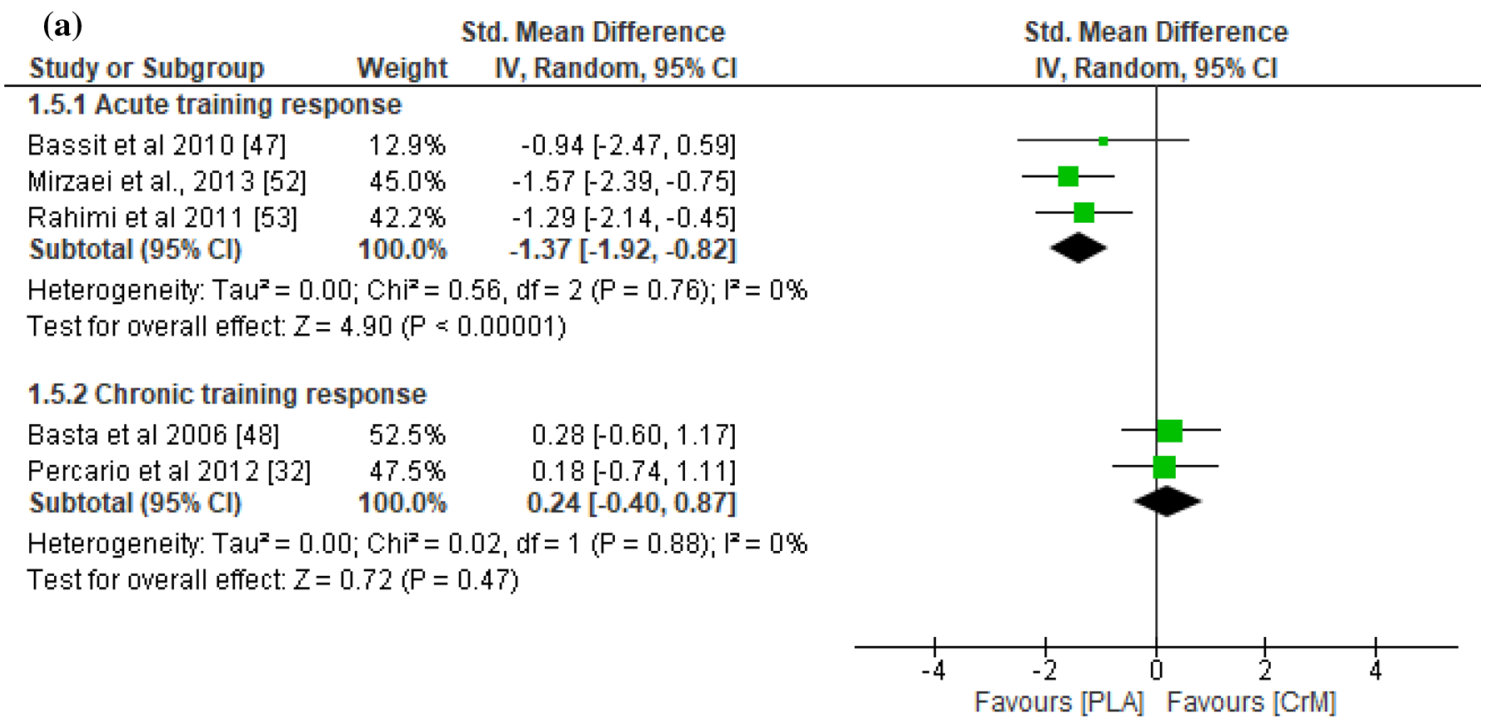

(b)

Study or Subgroup Weight $\quad$ IV, Random, $95 \% \mathrm{Cl}$

Std. Mean Difference IV, Random, $95 \% \mathrm{Cl}$

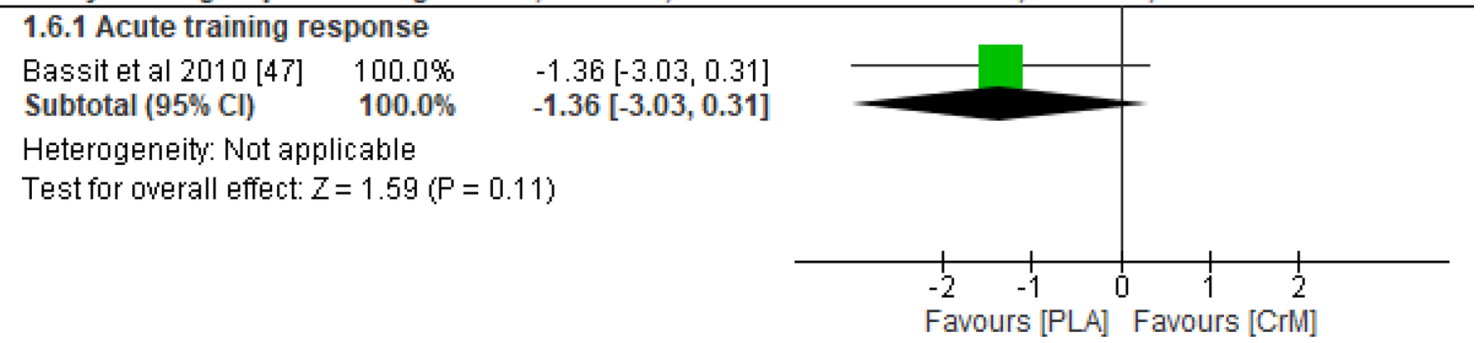

Fig. 4 Forest plot for oxidative stress at a 24 and $\mathbf{b} 48 \mathrm{~h}$ after the muscle-damaging protocol. $C r M$ creatine monohydrate group, $P L A$ placebo group

[28], and muscle force at $24 \mathrm{~h} \mathrm{[35]} \mathrm{and} 48 \mathrm{~h}$ [36]. When excluding these outliers, neither heterogeneity nor effect estimates were influenced for post-exercise muscle damage at $24 \mathrm{~h}\left(I^{2}=76 \%\right.$; SMD $\left.-0.08 ; p=0.45\right)$, oxidative stress at $24 \mathrm{~h}\left(I^{2}=0 \%\right.$; SMD $\left.-1.44 ; p<0.001\right)$, DOMS at $48 \mathrm{~h}$ $\left(I^{2}=14 \% ; \mathrm{SMD}-0.06 ; p=0.78\right)$, and muscle force at $48 \mathrm{~h}$ $\left(I^{2}=68 \%\right.$; SMD $\left.0.34 ; p=0.48\right)$. However, post-exercise muscle damage at $48 \mathrm{~h}$ changed from large to moderate for SMD ( $\mathrm{I}^{2}=76 \%$; SMD $\left.-0.65 ; \mathrm{p}=0.14\right)$, inflammatory markers at $24 \mathrm{~h}$ changed from large to moderate for heterogeneity and from large to moderate for SMD $\left(I^{2}=73 \%\right.$; SMD - 0.56; $p=0.23)$, inflammatory markers at $48 \mathrm{~h}$ changed from large to low for heterogeneity and from large to small for SMD $\left(I^{2}=0 \%\right.$; SMD $\left.-0.39 ; p=0.30\right)$, DOMS at $24 \mathrm{~h}$ changed from moderate to low for heterogeneity $\left(I^{2}=45 \%\right.$; SMD $\left.0.33 ; p=0.32\right)$, and muscle force at $24 \mathrm{~h}$ changed from large to low for heterogeneity $\left(I^{2}=0 \%\right.$; SMD $0.36 ; p=0.15)$.

\subsection{Risk of Bias}

On visual inspection, the funnel plots appeared relatively symmetrical and evenly distributed for muscle damage at $24 \mathrm{~h}$ post-exercise (electronic supplementary material [ESM]-1a), DOMS at 24 and $48 \mathrm{~h}$ post-exercise (ESM-2a and $2 \mathrm{~b}$, respectively), oxidative stress at $24 \mathrm{~h}$ post-exercise (ESM-3a), and muscle force measures at 24 and $48 \mathrm{~h}$ postexercise (ESM 4a and 4b). However, the studies appeared to congregate towards the top of the funnel plot for muscle damage at $48 \mathrm{~h}$ post-exercise (ESM-1b), inflammatory markers at 24 and $48 \mathrm{~h}$ post-exercise (ESM 5a and 5b, respectively), and oxidative stress at $48 \mathrm{~h}$ post-exercise (ESM-3b). Egger's test did not indicate publication bias for the muscle damage markers at $24 \mathrm{~h}(p=0.55)$ and $48 \mathrm{~h}$ post-exercise $(p=0.17)$, although we did not conduct this test for the other outcome measures because the number of studies was insufficient. 


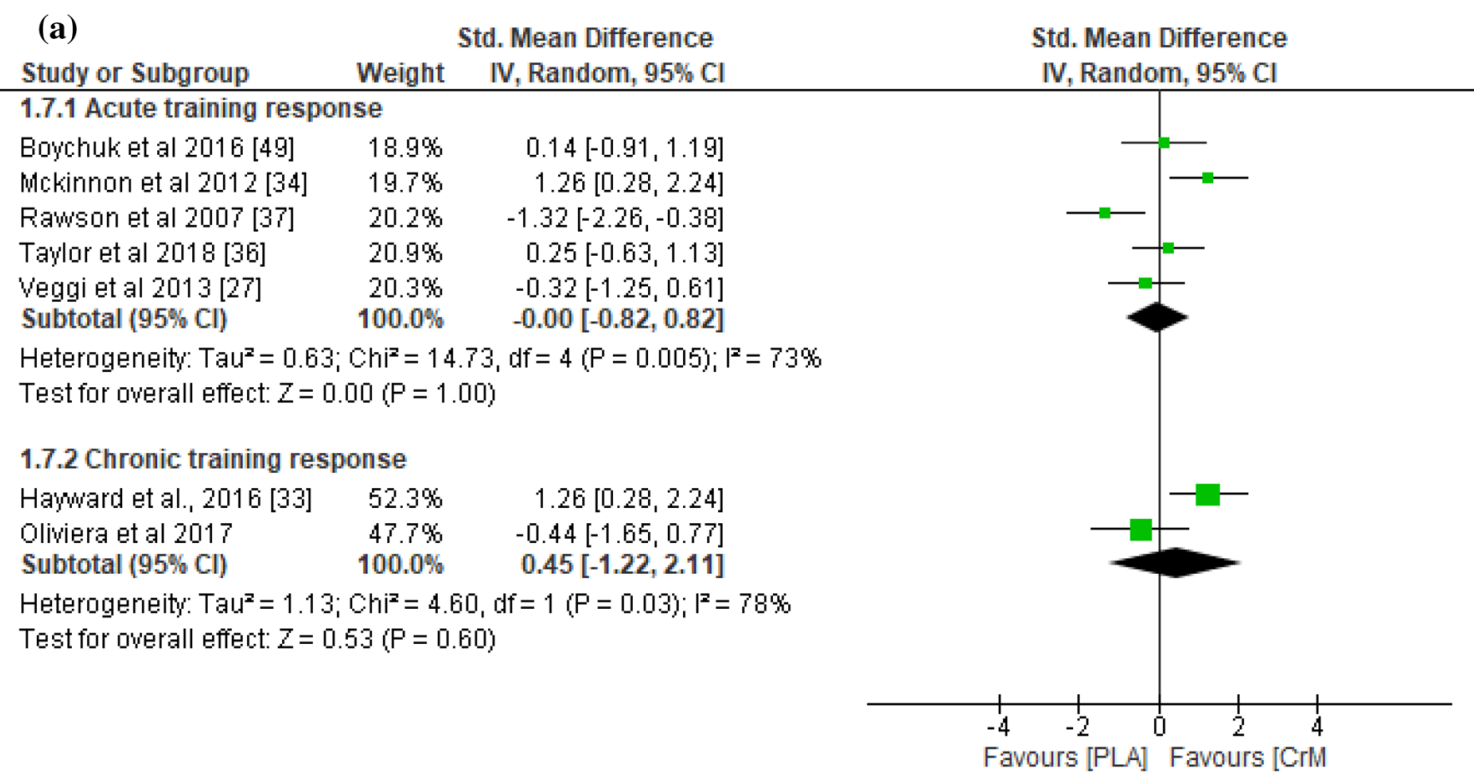

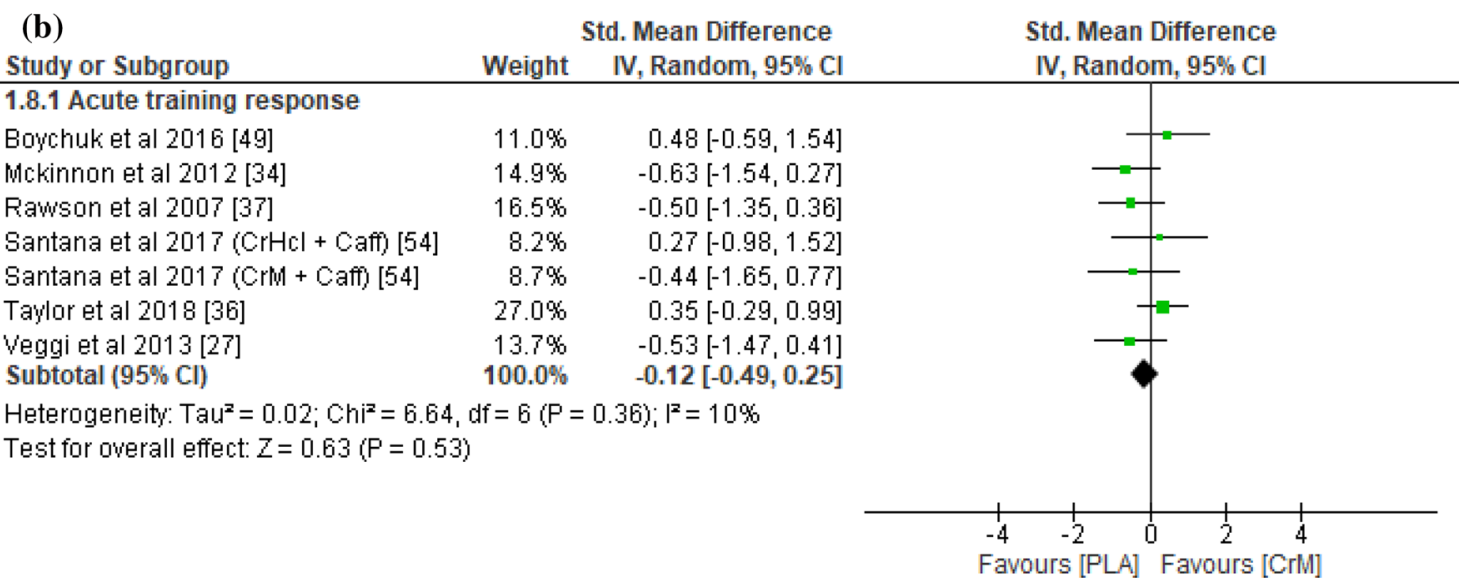

Fig. 5 Forest plot for delayed onset of muscle soreness at $\mathbf{a} 24$ and $\mathbf{b} 48 \mathrm{~h}$ after the muscle-damaging protocol. $C r M$ creatine monohydrate group, PLA placebo group

\section{Discussion}

This systematic review examined the effects of CrM supplements to reduce the signs and symptoms of EIMD. According to the meta-analysis, indirect markers of muscle damage and inflammatory and oxidative stress markers were lower in the CrM group after the muscle-damaging exercise as an acute training response, with large effect size calculations. Conversely, indirect muscle damage markers were higher in the CrM group as a chronic training response, also with large effect size calculations. Although no inter-group differences were identified for DOMS measures, the values appeared lower for the CrM group, with moderate effect size calculations, as an acute training response. There were no inter-group differences in muscle performance measures, with small effect size calculations for both acute and chronic training responses. Overall, there was some evidence that $\mathrm{CrM}$ reduced the level of EIMD as an acute training response, but exacerbated it for indirect muscle damage and inflammatory markers as a chronic training response, although this was dependent on the period of EIMD (i.e., 24-36-h or 48-90-h post-exercise). Furthermore, CrM did not appear to aid in the recovery of muscle performance measures following muscle-damaging exercises.

The findings from our meta-analysis were in line with the meta-analysis by Northeast and Clifford [24], whereby indirect muscle damage markers were only significantly lower for CrM at $48 \mathrm{~h}$ post-exercise, with minimal differences observed for DOMS and muscle force measures. Interestingly, muscle force measures showed a large effect size (SMD - 0.86) in the meta-analysis by Northeast and Clifford [24] at $24 \mathrm{~h}$ post-exercise, whereas our meta-analysis 


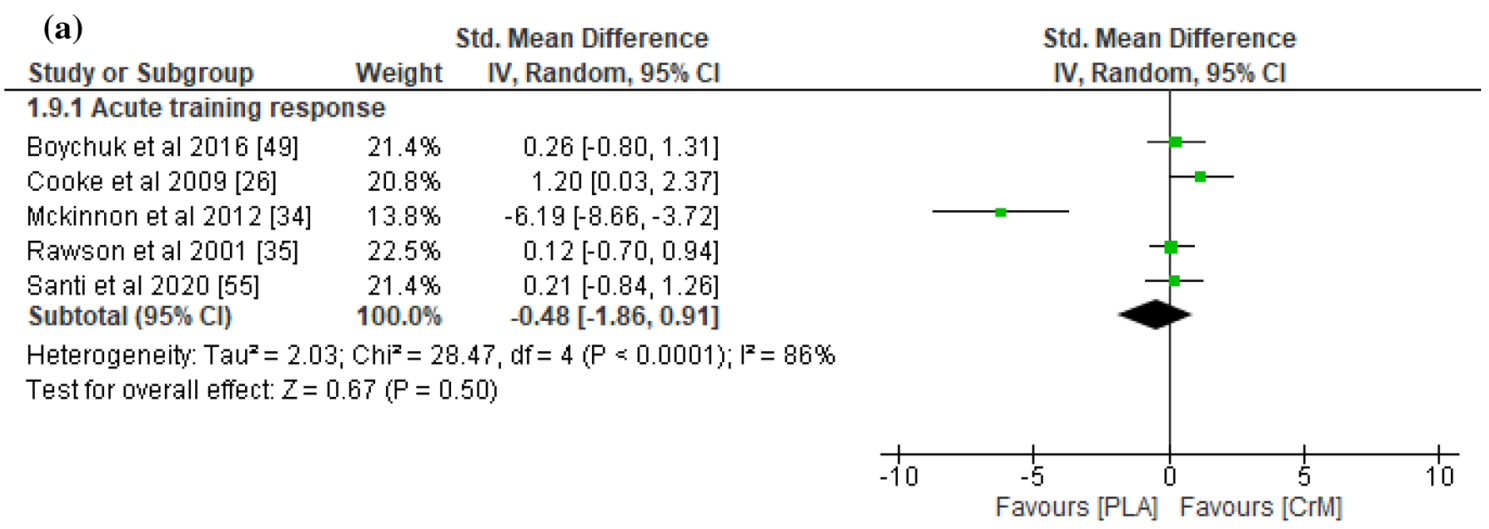

(b)

Std. Mean Difference

Study or Subgroup Weight IV, Random, $95 \% \mathrm{Cl}$
1.10.1 Acute training respons

Boychuk et al 2016 [49] $\quad 19.7 \%$ Cooke et al $2009[26] \quad 16.0 \%$ Mckinnon et al 2012 [34] $21.1 \%$ Rawson et al 2001 [35] $23.8 \%$ Santi et al $2020[55] \quad 19.4 \%$ Subtotal $(95 \% \mathrm{Cl}) \quad 100.0 \%$

$0.14[-0.91,1.19]$

$1.73[0.44,3.02]$

$-0.75[-1.71,0.22]$

$0.20[-0.62,1.02]$

$0.50[-0.57,1.56]$

$0.29[-0.41,0.99]$

Heterogeneity: $\mathrm{Tau}^{2}=0.36 ; \mathrm{Chi}^{2}=9.37, \mathrm{df}=4(\mathrm{P}=0.05) ; \mathrm{I}^{2}=57 \%$

Test for overall effect: $Z=0.81(P=0.42)$
Std. Mean Difference IV, Random, $95 \% \mathrm{Cl}$

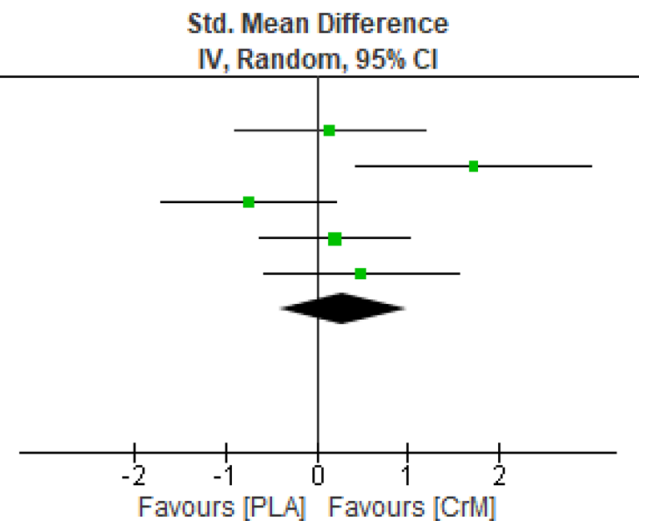

Fig. 6 Forest plot for muscle force measures at $\mathbf{a} 24$ and $\mathbf{b} 48 \mathrm{~h}$ after the muscle-damaging protocol. CrM creatine monohydrate group, $P L A$ placebo group

only showed a small effect size (SMD - 0.48) in the same direction. The discrepancy in data analysis may be due to the inclusion of the recently published study by Santi et al. [55], who reported a small effect size (SMD 0.21), but which was not included in the meta-analysis by Northeast and Clifford [24]. Furthermore, comparing our data on the oxidative stress and inflammatory markers to previous work is currently difficult because the current meta-analysis and systematic review is the first to examine the effects of CrM on these markers during periods of EIMD. Nonetheless, previous work has employed the same approach, by conducting meta-analysis to examine the ergogenic aids of supplements during periods of EIMD, but with plant extracts. Doma et al. [13] recently conducted a systematic review and meta-analysis of fruit-derived supplements on EIMD measures, showing significantly lower values for inflammation and oxidative stress with fruit-derived supplements than with placebo 24-48 $\mathrm{h}$ after the muscle-damaging exercises. However, their SMD values only ranged from 0.20 to 0.34 , which is substantially smaller than the SMD values of inflammation and oxidative stress identified in the current meta-analyses for CrM as an acute training response (SMD 0.91-1.79). In another recent meta-analysis that examined the effect of root plant supplements on EIMD measures [15], inflammation was significantly lower than with placebo conditions at 24-48 h post-exercise. However, their SMD values ranged from 0.09 to 0.34 , which again, are notably less than the SMDs of the current meta-analyses. Thus, CrM appears to provide greater protection against inflammation and oxidative stress than supplements derived from plants as an acute training response. These differences between meta-analyses may be attributed to the distinct biochemical constituents of CrM and plant-based extracts.

Several mechanisms have been proposed to explain how CrM ameliorates the signs and symptoms of EIMD, although they are not completely clear. First, a plethora of evidence demonstrates that muscle-damaging exercises increase inflammation, and this inflammatory response is believed to augment markers of EIMD via the secondary muscle damage response [1]. Furthermore, the elevation in inflammatory response also generates reactive oxygen species, which increases oxidative stress [57]. These processes cause further damage to already damaged and non-damaged muscle fibres, which accelerates myocyte membrane damage via peroxidation [58]. However, supplementation of $\mathrm{CrM}$ is believed to counteract increases in 
both inflammation and oxidative stress, which would limit further damage to skeletal muscle [59]. Our meta-analysis partly confirms this hypothesis, whereby the CrM group exhibited lower inflammatory and oxidative stress markers than the placebo group for up to $48 \mathrm{~h}$ post-exercise, with large effect sizes. However, we were unable to analyse the anti-inflammatory and antioxidant capacities of CrM because an insufficient number of studies examined these markers. Deminice and Jordao [25] showed that CrM supplementation decreased TBARS and increased total antioxidant capacity; however, this study was conducted in rats so further research is necessary to confirm the antiinflammatory and antioxidant roles and associated mechanisms of CrM in humans.

The current meta-analysis did not identify significant inter-group differences in DOMS between the CrM and placebo groups. One reason for this trend may be the subjectivity and limited inter-day reliability of the instruments used to measure DOMS [60], which would require a greater sample size to identify significant differences. Nonetheless, the values appeared smaller for the CrM group than for the placebo group, with a moderate effect size for up to $24 \mathrm{~h}$ postexercise. It has been suggested that the mechanical damage of the intermediate myofilaments activates group III and IV afferent nociceptors, resulting in symptoms of DOMS [2]. In the current systematic review, supplementation of CrM exhibited lower levels of indirect muscle damage, inflammation, and oxidative stress markers. Thus, we can assume that the antioxidant and anti-inflammatory capabilities of CrM reduced the activation of nociceptors, thereby minimising the symptoms of DOMS following muscle-damaging exercises.

Impaired muscle performance is a common occurrence during periods of EIMD. Possible explanations include alterations in the length of sarcomere caused by mechanical damage of muscle fibres, impaired excitation-contraction coupling, and influx of calcium concentrations, leading to prolonged deficits of muscular contractility [1]. However, the increase in intra-muscular phosphocreatine following the ingestion of CrM accelerates re-phosphorylation of adenosine triphosphate. This process sustains sarcoplasmic reticular calcium pump function by decreasing cytosolic calcium concentration [61], which is believed to enhance recovery of muscular function following the ingestion of $\mathrm{CrM}$. Interestingly, the current meta-analysis showed no inter-group differences between the $\mathrm{CrM}$ and placebo groups for muscle performance measures, with small effect size calculations. The limited effect of $\mathrm{CrM}$ on muscle performance could be attributed to the variety of methods used to measure muscle force (e.g., vertical jump height vs. isometric contractions) and the muscle groups assessed (e.g., knee extensors vs. elbow flexors). This results in a complex interaction of a number of different biomechanical and physiological factors influencing performance. Further, the limited number of studies assessing this specific outcome measure could be another reason for the absence of significant results. In fact, Doma et al. [13] also suggested similar confounders when they reported the lack of any differences in muscle performance measures with root plant supplements during periods of EIMD in their meta-analysis. Thus, more research is necessary to confirm the effects of $\mathrm{CrM}$ as a supplement to benefit the recovery of muscle strength. In this regard, the use of valid neuromuscular measures in low-complex tasks would be recommended to better isolate the effects of $\mathrm{CrM}$ on neuromuscular function.

Although the current meta-analysis showed that CrM may minimise the level of EIMD following muscle-damaging exercises as an acute training response (i.e., one bout of muscle-damaging exercises), greater levels of EIMD were found as a chronic training response (i.e., the last bout of muscle-damaging exercises from several weeks of training in conjunction with supplementation of $\mathrm{CrM}$ ). Furthermore, this reversed trend was observed by all studies included in this systematic review that examined chronic training responses, which strengthens the possibility that $\mathrm{CrM}$ could also exacerbate the level of EIMD depending on the method of delivery. This paradoxical effect was unexpected, given that studies typically implement $\mathrm{CrM}$ as a supplement to reduce markers of EIMD. However, the majority of authors of studies that examined the chronic training responses suspected that CrM might have augmented the level of EIMD to a greater extent than placebo because of enhanced training adaptations. For example, Kaviani et al. [32] suggested that the participants in their CrM group exhibited significantly greater CK measures than the placebo group after 8 weeks of resistance training with supplements because of an accelerated progression of resistance training intensity in the $\mathrm{CrM}$ group. Furthermore, the increase in intra-muscular phosphocreatine stores may have allowed for a higher training volume with the CrM group, resulting in greater damage to the muscles in a dose-response manner. Similar trends were also observed in the study by Brose et al. [50], who speculated that long-term CrM supplementation with several weeks of resistance training would increase total muscle creatine and fat-free mass, thereby augmenting the concentration of creatinine in the plasma. Thus, although CrM may provide protection against muscle damage in the short term following the first few training sessions, this trend may be reversed with longer-term supplementation and training. Possible strategies to ameliorate greater levels of EIMD as a chronic training response may be to consider a combination of oral supplements to manage EIMD, such as combining CrM with other supplements (e.g., herbal supplements, fruits, branched chain amino acids). Nonetheless, it is important to note that this heightened level of physiological stress may 
be necessary for enhanced adaptations to occur given that the $\mathrm{CrM}$ groups also exhibited greater training adaptations.

A number of issues need to be addressed in future research. First, more studies should consider assessing the bioavailability of CrM: $>70 \%$ of studies included in this systematic review did not consider this factor. This should be an essential component of these studies, given that the ergogenic effects of $\mathrm{CrM}$ for recovery reflect the absorption rate of $\mathrm{CrM}$, which in turn, is the most effective method of confirming the placebo effect. Second, studies reported only certain biomarkers to gain insight into the mechanisms contributing to the protective effects of $\mathrm{CrM}$ on the signs and symptoms of EIMD. Future studies should incorporate a range of biomarkers to develop a better understanding of muscle damage (including collagenase matrix metalloproteinase and B-cell lymphoma 2-associated athanogene 3) [62], anti-inflammatory and antioxidant effects of CrM during EIMD and proteostasis, and the potential ergogenic role of CrM for muscle recovery. Finally, although some of the studies included in this systematic review combined both males and females in their sample, whether sex affects the ergogenic effects of CrM during periods of EIMD remains unclear, warranting further research.

A number of limitations in this systematic review should be identified. First, several types of muscle-damaging protocols and participant characteristics were amalgamated metaanalytically, which may have impacted the degree of change in outcome measures. This is an important consideration because the level of EIMD is dependent on the training background [63], mode [64], and intensity [65] of exercise. Second, the rate of recovery with CrM supplementation appeared to vary between $24-36 \mathrm{~h}$ and $48-90 \mathrm{~h}$ post-exercise for each outcome measure, making precise recommendations difficult for each outcome measure. Third, the dosage of CrM was distinct between studies, also causing difficulty in providing exact recommendations on the amount of CrM required to optimise recovery following strenuous exercise. Thus, more research is necessary to improve recommendations on the dosage method for CrM supplementation and the time course recovery following strenuous exercises. Fourth, markers of muscle damage and oxidative stress may increase more than $90 \mathrm{~h}$ post-exercise in some cases [66], limiting the possibility of capturing a precise trend over time after strenuous exercises. Finally, we excluded all studies published in languages other than English, which may have introduced cultural bias.

\section{Conclusion}

Our systematic review and meta-analysis demonstrated a paradoxical effect of CrM supplementation, where the level of EIMD was reduced for several days after muscle-damaging exercises as an acute training response, but this trend was reversed as a chronic training response. Accordingly, coaches and athletes could consider incorporating $\mathrm{CrM}$ to aid in the acute recovery of strenuous training sessions, with the expectation that training-induced physiological stress and EIMD symptoms may be augmented following long-term use of CrM. However, further research is necessary to determine the ergogenic effects of $\mathrm{CrM}$ as a recovery supplement for muscular contractility during periods of EIMD.

Supplementary Information The online version contains supplementary material available at https://doi.org/10.1007/s40279-022-01640-z.

\section{Declarations}

Funding Open Access funding enabled and organized by CAUL and its Member Institutions. No sources of funding were used to assist in the preparation of this article.

Conflicts of Interest Kenji Doma, Akhilesh Kumar Ramachandran, Daniel Boullosa, and Jonathan Connor have no conflicts of interest that are directly relevant to the content of this article.

Availability of data and material Data will be provided upon request.

Code availability Not applicable.

Ethics approval Not applicable.

Consent to participate Not applicable.

Consent for publication Not applicable.

Author contributions KD wrote the first draft. AKR and JDC screened the abstracts and extracted the data from the included studies. AKR constructed the tables. AKR and JDC critically appraised the included studies. KD generated the forest plots. KD and DB analysed and interpreted the data. DB revised the original manuscript, and all authors read and approved the final manuscript.

Open Access This article is licensed under a Creative Commons Attribution 4.0 International License, which permits use, sharing, adaptation, distribution and reproduction in any medium or format, as long as you give appropriate credit to the original author(s) and the source, provide a link to the Creative Commons licence, and indicate if changes were made. The images or other third party material in this article are included in the article's Creative Commons licence, unless indicated otherwise in a credit line to the material. If material is not included in the article's Creative Commons licence and your intended use is not permitted by statutory regulation or exceeds the permitted use, you will need to obtain permission directly from the copyright holder. To view a copy of this licence, visit http://creativecommons.org/licenses/by/4.0/. 


\section{References}

1. Hyldahl RD, Hubal MJ. Lengthening our perspective: morphological, cellular, and molecular responses to eccentric exercise. Muscle Nerve. 2014;49(2):155-70.

2. Ebbeling CB, Clarkson PM. Exercise-induced muscle damage and adaptation. Sports Med. 1989;7(4):207-34.

3. Peake JM, Neubauer O, Della Gatta PA, Nosaka K. Muscle damage and inflammation during recovery from exercise. J Appl Physiol (1985). 2017;122(3):559-70.

4. Pyne DB. Exercise-induced muscle damage and inflammation: a review. Aust J Sci Med Sport. 1994;26(3-4):49-58.

5. Doma K, Deakin GB. The effects of strength training and endurance training order on running economy and performance. Appl Physiol Nutr Metab. 2013;38(6):651-6.

6. Doma K, Schumann M, Leicht AS, Heilbronn BE, Damas F, Burt D. The repeated bout effect of traditional resistance exercises on running performance across 3 bouts. Appl Physiol Nutr Metab. 2017;42(9):978-85.

7. Doma K, Schumann M, Sinclair WH, Leicht AS, Deakin GB, Hakkinen $\mathrm{K}$. The repeated bout effect of typical lower body strength training sessions on sub-maximal running performance and hormonal response. Eur J Appl Physiol. 2015;115(8):1789-99.

8. Hayter KJ, Doma K, Schumann M, Deakin G. The comparison of cold-water immersion and cold air therapy on maximal cycling performance and recovery markers following strength exercises. PeerJ. 2016;4: e1841. https://doi.org/10.7717/peerj.1841.

9. Doma K, Leicht A, Sinclair W, Schumann M, Damas F, Burt $\mathrm{D}$, et al. Impact of exercise-induced muscle damage on performance test outcomes in elite female basketball players. J Strength Cond Res. 2018;32(6):1731-8.

10. Khan MA, Moiz JA, Raza S, Verma S, Shareef MY, Anwer $\mathrm{S}$, et al. Physical and balance performance following exercise induced muscle damage in male soccer players. J Phys Ther Sci. 2016;28(10):2942-9.

11. Doma K, Deakin GB, Bentley DJ. Implications of impaired endurance performance following single bouts of resistance training: an alternate concurrent training perspective. Sports Med. 2017;47(11):2187-200.

12. Doma K, Deakin GB, Schumann M, Bentley DJ. Training considerations for optimising endurance development: an alternate concurrent training perspective. Sports Med. 2019;49(5):669-82.

13. Doma K, Gahreman D, Connor J. Fruit supplementation reduces indices of exercise-induced muscle damage: a systematic review and meta-analysis. Eur J Sport Sci. 2020;15:1-18.

14. Doma K, Gahreman D, Ramachandran AK, Singh U, Connor J. The effect of leaf extract supplementation on exercise-induced muscle damage and muscular performance: a systematic review and meta-analysis. J Sports Sci. 2021;19:1-17.

15. Doma K, Devantier-Thomas B, Gahreman D, Connor J. Selected root plant supplementation reduces indices of exercise-induced muscle damage: a systematic review and meta-analysis. Int $\mathbf{J}$ Vitam Nutr Res. 2020;16:1-21.

16. Lanhers C, Pereira B, Naughton G, Trousselard M, Lesage FX, Dutheil F. Creatine supplementation and upper limb strength performance: a systematic review and meta-analysis. Sports Med. 2017;47(1):163-73.

17. Farshidfar F, Pinder MA, Myrie SB. Creatine supplementation and skeletal muscle metabolism for building muscle mass- review of the potential mechanisms of action. Curr Protein Pept Sci. 2017;18(12):1273-87.

18. Volek JS, Duncan ND, Mazzetti SA, Staron RS, Putukian M, Gomez AL, et al. Performance and muscle fiber adaptations to creatine supplementation and heavy resistance training. Med Sci Sports Exerc. 1999;31(8):1147-56.

19. Willoughby DS, Rosene J. Effects of oral creatine and resistance training on myosin heavy chain expression. Med Sci Sports Exerc. 2001;33(10):1674-81.

20. Hespel P, Op't Eijnde B, Van Leemputte M, Urso B, Greenhaff PL, Labarque V, et al. Oral creatine supplementation facilitates the rehabilitation of disuse atrophy and alters the expression of muscle myogenic factors in humans. J Physiol. 2001;536(Pt 2):625-33.

21. Dangott B, Schultz E, Mozdziak PE. Dietary creatine monohydrate supplementation increases satellite cell mitotic activity during compensatory hypertrophy. Int J Sports Med. 2000;21(1):13-6.

22. Racette SB. Creatine supplementation and athletic performance. J Orthop Sports Phys Ther. 2003;33(10):615-21.

23. Rawson ES, Volek JS. Effects of creatine supplementation and resistance training on muscle strength and weightlifting performance. J Strength Cond Res. 2003;17(4):822-31.

24. Northeast B, Clifford T. The effect of creatine supplementation on markers of exercise-induced muscle damage: a systematic review and meta-analysis of human intervention trials. Int J Sport Nutr Exerc Metab. 2021;31(3):276-91.

25. Deminice R, Jordao AA. Creatine supplementation reduces oxidative stress biomarkers after acute exercise in rats. Amino Acids. 2012;43(2):709-15.

26. Nomura A, Zhang M, Sakamoto T, Ishii Y, Morishima Y, Mochizuki M, et al. Anti-inflammatory activity of creatine supplementation in endothelial cells in vitro. Br J Pharmacol. 2003;139(4):715-20.

27. Cooke MB, Rybalka E, Williams AD, Cribb PJ, Hayes A. Creatine supplementation enhances muscle force recovery after eccentrically-induced muscle damage in healthy individuals. J Int Soc Sports Nutr. 2009;2(6):13.

28. Veggi KF, Machado M, Koch AJ, Santana SC, Oliveira SS, Stec MJ. Oral creatine supplementation augments the repeated bout effect. Int J Sport Nutr Exerc Metab. 2013;23(4):378-87.

29. Wang CC, Fang CC, Lee YH, Yang MT, Chan KH. Effects of 4-week creatine supplementation combined with complex training on muscle damage and sport performance. Nutrients. 2018;10(11):1640.

30. Claudino JG, Mezencio B, Amaral S, Zanetti V, Benatti F, Roschel $\mathrm{H}$, et al. Creatine monohydrate supplementation on lower-limb muscle power in Brazilian elite soccer players. J Int Soc Sports Nutr. 2014;11:32.

31. Fernandez-Landa J, Fernandez-Lazaro D, Calleja-Gonzalez J, Caballero-Garcia A, Cordova A, Leon-Guereno P, et al. Longterm effect of combination of creatine monohydrate plus betahydroxy beta-methylbutyrate (HMB) on exercise-induced muscle damage and anabolic/catabolic hormones in elite male endurance athletes. Biomolecules. 2020;10(1):140.

32. Kaviani M, Abassi A, Chilibeck PD. Creatine monohydrate supplementation during eight weeks of progressive resistance training increases strength in as little as two weeks without reducing markers of muscle damage. J Sports Med Phys Fitness. 2019;59(4):608-12.

33. Percario S, Domingues SP, Teixeira LF, Vieira JL, de Vasconcelos F, Ciarrocchi DM, et al. Effects of creatine supplementation on oxidative stress profile of athletes. J Int Soc Sports Nutr. 2012;9(1):56.

34. Hayward S, Wilborn CD, Taylor LW, Urbina SL, Outlaw JJ, Foster $\mathrm{CA}$, et al. Effects of a high protein and omega-3-enriched diet with or without creatine supplementation on markers of soreness and inflammation during 5 consecutive days of high volume resistance exercise in females. J Sports Sci Med. 2016;15(4):704-14.

35. McKinnon NB, Graham MT, Tiidus PM. Effect of creatine supplementation on muscle damage and repair following 
eccentrically-induced damage to the elbow flexor muscles. J Sports Sci Med. 2012;11(4):653-9.

36. Rawson ES, Gunn B, Clarkson PM. The effects of creatine supplementation on exercise-induced muscle damage. J Strength Cond Res. 2001;15(2):178-84.

37. Taylor BA, Panza G, Ballard KD, White CM, Thompson PD. Creatine supplementation does not alter the creatine kinase response to eccentric exercise in healthy adults on atorvastatin. J Clin Lipidol. 2018;12(5):1305.

38. Rawson ES, Conti MP, Miles MP. Creatine supplementation does not reduce muscle damage or enhance recovery from resistance exercise. J Strength Cond Res. 2007;21(4):1208-13.

39. Moher D, Liberati A, Tetzlaff J, Altman DG, Group P. Preferred reporting items for systematic reviews and meta-analyses: the PRISMA statement. BMJ. 2009;21(339): b2535.

40. Kamper SJ, Moseley AM, Herbert RD, Maher CG, Elkins MR, Sherrington C. 15 years of tracking physiotherapy evidence on PEDro, where are we now? Br J Sports Med. 2015;49(14):907-9.

41. Johnston R, Doma K, Crowe M. Nicotine effects on exercise performance and physiological responses in nicotine-naive individuals: a systematic review. Clin Physiol Funct Imaging. 2018;38(4):527-38.

42. Higgins JPT, Green S. Cochrane handbook for systematic reviews of interventions version. Chichester: Wiley; 2011.

43. Moeyaert M, Ugille M, Beretvas N, Ferron J, Bunuan R, Van den Noortgate W. Methods for dealing with multiple outcomes in meta-analysis: a comparison between averaging effect sizes, robust variance estimation and multilevel meta-analysis. Int J Soc Res Method. 2017;20(6):559-72.

44. Cohen J. Statistical power analysis for the behavioral sciences. Hillsdale: Lawrence Erlbaum Associates; 1988.

45. Atashak S, Jafari A. Effect of short-term creatine monohydrate supplementation on indirect markers of cellular damage in young soccer players. Sci Sports. 2012;27(2):88-93.

46. Bassit RA, Curi R, Costa Rosa LF. Creatine supplementation reduces plasma levels of pro-inflammatory cytokines and PGE2 after a half-ironman competition. Amino Acids. 2008;35(2):425-31.

47. Bassit RA, Pinheiro CH, Vitzel KF, Sproesser AJ, Silveira LR, Curi R. Effect of short-term creatine supplementation on markers of skeletal muscle damage after strenuous contractile activity. Eur J Appl Physiol. 2010;108(5):945-55.

48. Basta P, Skarpanska-Steinborn A, Placzynska-Szczesniak L. Creatine supplmenetation and parameters of exercise-induced oxidative stress after a standard rowing test. Stud Physical Cult Tour. 2006;13(1):17-23.

49. Boychuk KE, Lanovaz JL, Krentz JR, Lishchynsky JT, Candow DG, Farthing JP. Creatine supplementation does not alter neuromuscular recovery after eccentric exercise. Muscle Nerve. 2016;54(3):487-95.

50. Brose A, Parise G, Tarnopolsky MA. Creatine supplementation enhances isometric strength and body composition improvements following strength exercise training in older adults. J Gerontol A Biol Sci Med Sci. 2003;58(1):11-9.

51. Machado M, Pereira R, Sampaio-Jorge F, Knifis F, Hackney A. Creatine supplementation: effects on blood creatine kinase activity responses to resistance exercise and creatine kinase activity measurement. Braz J Pharm Sci. 2009;45(4):751-7.
52. Mirzaei B, Fahmani-Nia F, Salehi Z, Rahimi R. Effects of creatine monohydrate supplementation on oxidative DNA damage and lipid peroxidation induced by acute incremental exercise to exhaustion in wrestlers. Kinesiology. 2013;45(1):30-40.

53. Rahimi R. Creatine supplementation decreases oxidative DNA damage and lipid peroxidation induced by a single bout of resistance exercise. J Strength Cond Res. 2011;25(12):3448-55.

54. Santana JO, Franca E, Madureira D, Rodrigues B, Caperuto EC. Combined effect of creatine monohydrate or creatine hydrochloride and caffeine supplementation in runners' performance and body composition. Revista Brasileira de Prescrição e Fisiologia do Exercício. 2017;11:844-54.

55. Santi MC, Galan BS, Terrazas SI, De Carvalho FG, Viera TS, Silveira GC, et al. Effect of creatine supplementation on muscle damage markers and physical performance in volleyball athletes. Cultura, Ciencia y Deporte. 2020;15(45):377-85.

56. Santos RV, Bassit RA, Caperuto EC, Costa Rosa LF. The effect of creatine supplementation upon inflammatory and muscle soreness markers after a 30km race. Life Sci. 2004;75(16):1917-24.

57. Aoi W, Naito Y, Takanami Y, Kawai Y, Sakuma K, Ichikawa H, et al. Oxidative stress and delayed-onset muscle damage after exercise. Free Radic Biol Med. 2004;37(4):480-7.

58. Mastaloudis A, Morrow JD, Hopkins DW, Devaraj S, Traber MG. Antioxidant supplementation prevents exercise-induced lipid peroxidation, but not inflammation, in ultramarathon runners. Free Radic Biol Med. 2004;36(10):1329-41.

59. Lawler JM, Barnes WS, Wu G, Song W, Demaree S. Direct antioxidant properties of creatine. Biochem Biophys Res Commun. 2002;290(1):47-52.

60. Lau WY, Muthalib M, Nosaka K. Visual anaolg scale and pressure pain threshold for delayed onset muscle soreness assessment. J Musculoskelet Pain. 2013;21(4):320-6.

61. Korge P, Byrd SK, Campbell KB. Functional coupling between sarcoplasmic-reticulum-bound creatine kinase and $\mathrm{Ca}(2+)$ ATPase. Eur J Biochem. 1993;213(3):973-80.

62. Boullosa D, Dragutinovic B, Deutsch JP, Held S, Donath L, Bloch $\mathrm{W}$, et al. Acute and delayed effects of time-matched very short "All Out" efforts in concentric vs. eccentric cycling. Int J Environ Res Public Health. 2021;18(15):7968.

63. Newton MJ, Morgan GT, Sacco P, Chapman DW, Nosaka K. Comparison of responses to strenuous eccentric exercise of the elbow flexors between resistance-trained and untrained men. J Strength Cond Res. 2008;22(2):597-607.

64. Nieman DC, Luo B, Dreau D, Henson DA, Shanely RA, Dew $\mathrm{D}$, et al. Immune and inflammation responses to a 3-day period of intensified running versus cycling. Brain Behav Immun. 2014;39:180-5.

65. Doma K, Deakin GB. The acute effects intensity and volume of strength training on running performance. Eur J Sport Sci. 2014;14(2):107-15.

66. Cakir-Atabek H, Dokumaci B, Aygun C. Strength loss after eccentric exercise is related to oxidative stress but not muscle damage biomarkers. Res Q Exerc Sport. 2019;90(3):385-94. 


\section{Authors and Affiliations}

\section{Kenji Doma ${ }^{1}$ - Akhilesh Kumar Ramachandran ${ }^{2} \cdot$ Daniel Boullosa $^{1,3} \cdot$ Jonathan Connor $^{1}$}

$\triangle$ Kenji Doma

kenji.doma@jcu.edu.au

1 James Cook Drive, Rehabilitation Sciences Building,

College of Healthcare Sciences, Sports and Exercise

Science, James Cook University, Douglas, QLD QLD481, Australia

2 Sports Dynamix Private Limited, Chennai, India
3 Federal University of Mato Grosso, Mato Grosso, Brazil 